\title{
A Theoretical Foundation for Timing Synchronous Systems Using Asynchronous Structures
}

\author{
RAMY N. TADROS and PETER A. BEEREL, University of Southern California
}

\begin{abstract}
Timing of synchronous systems is an everlasting stumbling block to the booming demands for lower power consumption and higher operation speeds in the electronics industry. This hardship is aggravated by the growing levels of variability in state-of-the-art silicon dimensions and in other beyond-CMOS technologies. Although some designers continue to strongly believe in the performance advantages of being fully synchronous, others have radically shifted toward extremely robust delay-insensitive domains. Targeting a different compromise of both performance and robustness, this article provides sufficient conditions for an asynchronous system to be able to generate the periodic signals necessary for the timing of a fully synchronous system and highlights a specific hierarchical clocking structure that with a single tunable delay satisfies these conditions. Using an asynchronous clock distribution network benefits from both the natural robustness of asynchronous structures and the advantageous performance of synchronous clocking.
\end{abstract}

CCS Concepts: • Hardware $\rightarrow$ Asynchronous circuits; Static timing analysis; Superconducting circuits; - Software and its engineering $\rightarrow$ Petri nets;

Additional Key Words and Phrases: Asynchronous circuits, synchronous systems, timing analysis, clocking, Petri nets, marked graphs, superconducting electronics, single flux quantum

ACM Reference format:

Ramy N. Tadros and Peter A. Beerel. 2020. A Theoretical Foundation for Timing Synchronous Systems Using Asynchronous Structures. ACM Trans. Des. Autom. Electron. Syst. 25, 2, Article 16 (January 2020), 28 pages. https://doi.org/10.1145/3373355

\section{INTRODUCTION}

Amid the portable electronics generation, on the verge of the IoT era, electronics designers are challenged by the upsurge of the market demands for low power consumption and higher operation speeds. Timing of synchronous systems is arguably one of the hardest obstacles to answering these demands. The power and performance cost of delivering a clocking signal to every clock sink in the chip is considerable when compared to the cost of performing the logical operations themselves. This is exacerbated with unprecedented levels of variability in state-of-the-art silicon dimensions and in other beyond-CMOS technologies [16].

The research is based upon work supported by the Office of the Director of National Intelligence (ODNI), Intelligence Advanced Research Projects Activity (IARPA), via the U.S. Army Research Office grant W911NF-17-1-0120. The views and conclusions contained herein are those of the authors and should not be interpreted as necessarily representing the official policies or endorsements, either expressed or implied, of the ODNI, IARPA, or the U.S. Government. The U.S. Government is authorized to reproduce and distribute reprints for Governmental purposes not withstanding any copyright notation herein. Authors' address: R. N. Tadros and P. A. Beerel, University of Southern California (USC), 3740 McClintock Avenue, Los Angeles, CA 90089; emails: \{rtadros, pabeerel\}@usc.edu.

Permission to make digital or hard copies of all or part of this work for personal or classroom use is granted without fee provided that copies are not made or distributed for profit or commercial advantage and that copies bear this notice and the full citation on the first page. Copyrights for components of this work owned by others than ACM must be honored. Abstracting with credit is permitted. To copy otherwise, or republish, to post on servers or to redistribute to lists, requires prior specific permission and/or a fee. Request permissions from permissions@acm.org.

(C) 2020 Association for Computing Machinery.

1084-4309/2020/01-ART16 \$15.00

https://doi.org/10.1145/3373355 
In synchronous systems, designing clock distribution networks (CDNs) has never been a trivial task $[9,12,29]$. Foreseeing the growing uncertainty in circuit parameters, the asynchronous community has offered more robust solutions that are more naturally tolerant to variability [3]. For example, many designers have resorted to globally asynchronous locally synchronous systems as a middle-ground compromise $[1,2,8,19]$. Other researchers, assuming utter uncertainty, proposed the use of extremely robust delay-insensitive circuits [7, 17, 30]. The work of Cortadella et al. [5] suggested the use of a ring oscillator (RO) instead of the traditional phase-locked loop (PLL) as a more variation-tolerant clocking scheme in which the RO drives a zero-skew tree to all clock sinks. Argo [18] uses asynchronous on-chip routers organized in a 2-D mesh network. These routers are interfaced to the fully synchronous processing cores, which are mesochronously clocked, using asynchronous ripple first-input first-output (FIFO) buffers. In other words, Argo is an example of a work that used asynchronous structures-the routers of the NoC to control a synchronously constrained environment-time-division multiplexed network interfaces that are synchronously interfaced to the fully synchronous cores. The work of Cortadella et al. [4] proposed an asynchronous structure that synchronizes a network of oscillators on the same chip. The work of Tadros and Beerel $[31,32]$ proposed the use of a hierarchy of interconnected asynchronous oscillators to provide the clocking signals directly to the relatively large number of clock sinks present in gate-level pipelined superconducting electronics.

This article provides a foundation for such practical asynchronous CDNs. In particular, we state the sufficient conditions for an asynchronous system to provide periodic signals with well-defined clock skews for a large number of clock sinks. To illustrate the value of these conditions, we highlight a specific hierarchical clocking structure that with a single tunable delay satisfies these conditions [33]. The clocking structure benefits from both the natural robustness of asynchronous structures as it adapts to the worst-case delay of any local cycle and the advantageous performance of synchronous clocking.

We model these systems using timed marked graphs (MGs) from Petri net (PN) theory [25] and provide sufficient conditions for asynchronous structures to provide the clocking signals necessary for the clock sinks in a synchronous system. This is in contrast to the Argo system [18], for example, where they interface the asynchronous circuits to synchronous cores but do not use them to do the clocking; rather, they use a fully synchronous clock source. Other works have analyzed the performance and periodicity of timed MGs $[6,23,26]$, but none focused on identifying a class of MGs that can model a practical CDN.

We hereby list the contributions of this work:

- This article is the first to formalize the notion of an asynchronous clock distribution network $(\mathrm{ACDN})$ that benefits from the adaptability of asynchronous circuits while still being useful for clocking a synchronous circuit.

- It suggests and proves a unique and practical set of sufficient conditions that guarantees an asynchronous circuit can be used as an ACDN, illustrating its benefits in the domain of digital superconductive electronics.

- It also highlights some mistakes in the literature regarding the necessary conditions for periodicity in MGs.

The remainder of this article is organized as follows. First, Section 3 provides a problem formulation along with an overview of related work. Section 2 then briefly explains some terms and nomenclature used in this article, whereas the formal definitions are included in the appendix. Section 4 introduces the ACDN theoretical foundation, and then the main theoretical work and its derivations are presented in Section 5. After that, Section 6 compares this work to related previous literature. Finally, Section 7 provides a discussion, and Section 8 presents our conclusion. 


\section{BRIEF SUMMARY OF DEFINITIONS}

In this section, we provide a brief and informal summary of definition and nomenclature (a formal and more detailed version with clarifying examples is provided in Appendix A):

Timed PN components. $p_{n}$ denotes the $n t h$ place, and $m_{n}$ is the initial number of markings in $p_{n}$. $t_{n}$ denotes the $n t h$ transition, and $\delta_{n}$ is the delay associated with $t_{n}$. The sources set $\mathcal{S}$ is defined such that a transition $t_{s} \in \mathcal{S}$ if it is enabled initially.

Marked graph. An MG is a PN such that each place $p$ has exactly one input transition and exactly one output transition [25].

Transition firing. $\tau_{n}^{(i)}$ is the time at which transition $t_{n}$ initiates its $i t h$ firing. Note that for sources, we set $\tau_{s}^{(0)}=0$.

Directed paths. A directed path $h$ is a sequence of alternating transitions and places; we define its delay $\Delta(h)$ as the sum of the delays in the transitions forming the path. A simple directed path is where all transitions are distinct. $\mathcal{H}\left(t_{u}, t_{v}\right)$ is the set of all possible directed paths from $t_{u}$ to $t_{v}$. A third argument, $m$, to $\mathcal{H}$ means that the number of tokens along this path is limited to $m$.

Directed circuits. A loop is a directed path that starts and ends at the same transition. A directed circuit is a loop with distinct transitions and places. We let the set $C$ denote the set of all directed circuits in the PN and let $M\left(C_{k}\right)$ denote the total number of tokens in the places of the $k$ th directed circuit, $C_{k}$.

Cycle time. We define the cycle time as the mean time to complete a firing sequence leading to the firing of each transition exactly once.

\section{PROBLEM FORMULATION AND RELATED WORKS}

In a synchronous system, each clock sink is expected to receive a clocking signal in every time period known as the cycle time [29]. Moreover, the difference in clock signal arrival time between any two clock sinks (i.e., the clock skew) should be well defined [9]. Conventionally, synchronous systems are designed to keep the skew value between any two sinks constant and ideally set to zero, although nothing is special about this particular value [10]. Additionally, the system usually starts with a transitional period that we call the warming-up period $[13,15]$ until the timing source settles and the system then enters a periodic steady state.

For any CDN, the desired timing of all clock sinks can be represented as follows:

$$
\tau_{n}^{(i)}=T_{u p}+T_{\text {skew }}+i \cdot T_{\text {sys }}, \quad \forall t_{n} \in \mathcal{T}, \forall i \geq i_{u p},
$$

where $\tau_{n}^{(i)}$ is is the ith time occurrence of the clock signal at the $n t h$ clock sink $t_{n}$, where $\mathcal{T}$ is the set of all clock sinks. $T_{\text {sys }}$ is the system cycle time and $T_{\text {skew }}$ is the clock skew value at the clock sink, $t_{n}$, with respect to a common reference. Ignoring variations and asymmetries, this skew should equal zero in zero-skew systems. $T_{u p}$ is the warming-up period, and $i_{u p}$ represents the warming-up occurrences before the system enters a periodic steady state $[13,15]$.

It should be emphasized that the value of $T_{\text {skew }}$ should be well defined for every transition. In other words, the clock path resulting in the nominal value of $T_{\text {skew }}$ should be known during the design phase. This is to guarantee a safe operation without any setup or hold violations that must be checked during design using some form of a static timing analysis (STA) [34]. It should also be noted that the periodic property in (1) is a desirable characteristic for any synchronous system. Otherwise, the synchronous datapath has to account for a time-varying clock period that is undesirable. Interestingly, the authors of Kasapaki et al. [18] were forced to address this problem designing an asynchronous NoC interfaced to synchronous cores. Furthermore, it is desirable for the cycle time, $T_{s y s}$, to be independent from the size of the clock network (i.e., the number of sinks). This is in contrast to the ACDN proposed in Tadros and Beerel [31], whose cycle time 
grew with the number of clock sinks. The works of Hulgaared et al. [15], Hua and Manohar[13], and Nielsen and Kishinevsky [26] have well understood the not-straightforward nature of asynchronous structures, especially regarding their periodicity. Using their respective mathematical approaches, they have deciphered the periodicity problem and provided methods and algorithms for finding the timing parameters and describing the timing behavior of asynchronous structures while making the least amount of assumptions. Nevertheless, since they were analyzing arbitrary MGs, their contributions were generic and they did not delve into conditions that guaranteed more restrictive forms of periodicity.

The work with the closest ties to our work is that of McGee and Nowick [23]. Although their focus was on practical algorithms to calculate time separation of events (TSE), they identified a sub-class of MGs similar to those proposed in this article with similar properties. However, the conditions of their MG class are not quite the same as the ones proposed in this work (see Section 6.2). In addition, this work studies the case when it is not guaranteed for the source to be in a critical circuit, which is essential for an asynchronous CDN (see Section 5.2). Moreover, this work presents a more formal approach toward its conclusions. More detailed insights and comparisons regarding these works are presented in Section 6.2.

The key contribution of this work is that we develop a specific hierarchical clocking structure and an associated class of timed MG models that can force the system's periodicity to strictly equal 1 as in (1) and the warming-up period $T_{u p}$ to equal zero, and, more crucially, to strictly define the skew value of every transition with respect to a certain reference. Instead of analyzing arbitrary networks and building algorithms to describe the behavior of a given graph as in other works [13, $15,23,26]$, our work provides design constraints for asynchronous structures that can be used as practical CDNs that are scalable and easily tuned for synchronous systems. In other words, our contribution is in graph design rather than graph analysis.

\section{SUFFICIENT CONDITIONS FOR ACDNS}

In this article, we provide sufficient conditions for ACDNs. Note that Appendix A recites the definitions and the nomenclature used in this document. Moreover, Appendix A explains in detail the basic concepts of MGs such as transition, place, and path using the MG example shown later in Figure 6. Informally, PNs are a graphical and mathematical modeling tool. A MG is a PN with no choice-that is, there is no event in an MG that triggers one subset of events rather than another subset. It should also be noted that we choose timed MGs (see Definition 7) to model the CDNs, as it is natural for clock delay elements to be modeled as transitions with a specific time delay with no choice. Moreover, clock delay elements do not have memories to allow more than one token per place, which guarantees safeness (see Definition 13). Regarding our choice of using deterministic time delay, this is for simplicity and to establish the main timing characteristics for a theoretical foundation. Practically, the time delays shall be random variables due to jitter and other uncertainties, and the timing characteristics shall possess a stochastic aspect as well. This is discussed in Section 7.

Definition 1. ACDNs are systems that can be modeled as timed MGs whose transitions firings follow the property in (1). ACDNs can thus be used as a CDN for a synchronous system.

In particular, if the MG model of an asynchronous system satisfies the following conditions:

- does not have always-enabled-transitions (i.e., with no external inputs),

- is live and safe,

- has a single token per directed circuit, and

- has a single source, and this source belongs to a critical circuit (see Definition 29), 


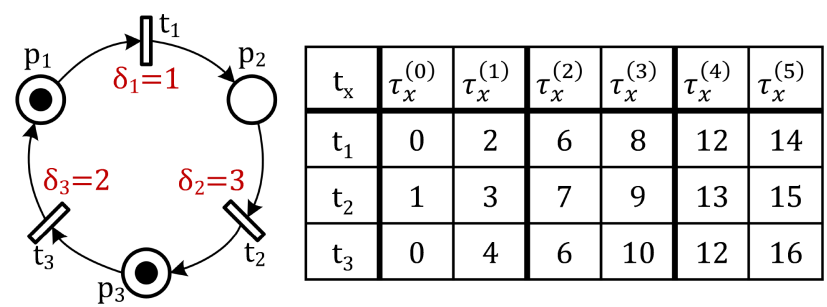

Fig. 1. An example of a MG with two sources and more than a token per circuit. $T_{s y s}=\frac{\Delta(C)}{M(C)}=\frac{6}{2}=3$.

then the system is an ACDN. In other words, all transitions in this system maintain the property in (1) and can thus be used to provide the clocking signals for a synchronous system without the need for an external input clock.

Specifically, the first condition excludes systems with externally generated clock signals. The second ensures the periodicity of (1). The third ensures that the clock period does not change from one firing occurrence to another-that is, the system periodicity equals 1 [13] (see Section 6.2). The last condition guarantees that the value of $T_{u p}$ equals zero and that the skew value is well defined for every clock sink. Section 7 discusses these statements in more detail and connects them to the theorems presented in this article.

In an attempt to clarify the intuition of our work and to further motivate the significance of those constraints, Figure 1 shows an MG example that violates two of the constraints: It has a directed circuit with more than one token, and it has more than one source. The table next to the diagram shows the first six transitions firings. The transitions firing cannot be described using (1) because the firing period oscillates. Focusing on $t_{1}$, for instance, substituting into (1) from the table, we get that $\tau_{1}^{(i)}=3 i$. However, this only works for even values of $i$; that is because its period oscillates between 2 and 4 . Such period oscillation is an undesirable property for a clocking signal of a synchronous system as discussed earlier in Section 3.

\section{THEORY}

In this section, we prove that the conditions mentioned previously are sufficient for an asynchronous system to guarantee the property in (1) enabling their use as a CDN for synchronous circuits. The full mathematical derivations are in Appendix B.

\subsection{Firing Period}

In this section, we formalize that under the constraints stated in Section 4, the asynchronous system satisfies the timing property in (1) and can thus be used as a CDN for a synchronous system.

Definition 2. MG-L is a live and safe MG [25].

Definition 3. MG-T is an MG with exactly one token in every directed circuit. Formally,

$$
\text { An MG is MG-T if } M(C)=1, \forall C \in C \text {. }
$$

Definition 4. MG-S is an MG with a single source. Formally,

$$
\text { An MG is MG-S if }|\mathcal{S}|=1 \text {, subject to } \mathcal{S}=\left\{t_{s}\right\} \text {. }
$$

Definition 5. MG-SC is an MG-S whose source belongs to a critical circuit (see Definition 29). Formally,

$$
\text { An MG-S is MG-SC if } C\left(t_{s}\right) \cap \arg \max _{C_{k} \in C}\left(\frac{\Delta\left(C_{k}\right)}{M\left(C_{k}\right)}\right) \neq \emptyset \text {. }
$$




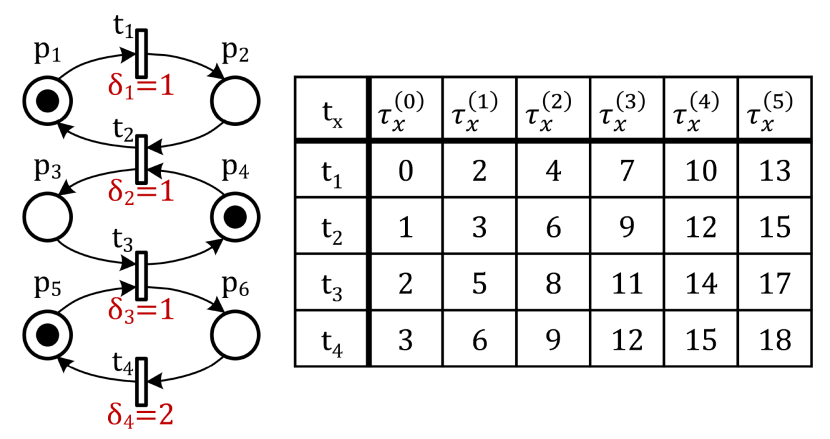

Fig. 2. An example of an MG whose source does not belong to a critical circuit. $T_{s y s}=\max _{C \in C}\left(\frac{\Delta(C)}{M(C)}\right)=$ $\frac{3}{1}=3$.

\section{Theorem 1}

In an MG-LTSC, $\forall t_{n} \in \mathcal{T}, \forall i \geq 0$,

$$
\tau_{n}^{(i)}=\left(\max _{h \in \hat{\mathcal{H}}\left(t_{s}, t_{n}, 0\right)} \Delta(h)\right)+i \cdot T_{\text {sys }} .
$$

In other words, given an MG-LTSC, the ith firing of any transition $t_{n}$ is periodic with a period of $T_{\text {sys }}$ and has a constant delay shift equal to the maximum zero tokens path from the source to $t_{n}$. Note that $\Delta(h)$ is the delay along a path $h$, and $\hat{\mathcal{H}}\left(t_{s}, t_{n}, 0\right)$ is the set if all zero tokens directed paths from the source $t_{s}$ to the transition $t_{n}$. More formal definitions are provided in Appendix A. In addition, note that a similar result with more strict conditions is argued for in McGee and Nowick [23]. The complete and formal proof of our theorem is in Appendix B.3.

\section{Violating examples:}

(1) The example of Figure 1 that is discussed after Theorem 4 violates both the $\mathrm{T}$ and the $\mathrm{S}$ constraints; it has a directed circuit with more than one token, and it has more than one source. Same as for Theorem 4, since the firing period oscillates, the transitions firing cannot be described using (5). It should be observed here that the difference between Theorems 4 and 1 is that the former defines the periodicity (the $i \cdot T_{\text {sys }}$ part of (1)), whereas the latter, which possesses the same periodicity, defines the constant delay shift $\left(T_{\text {skew }}\right.$ in (1)) that is specific to every transition in the graph.

(2) In an attempt to further clarify the intuition of Theorem 1, Figure 2 shows an MG example that violates the SC constraint; its source does not belong to a critical circuit. The source does belong to only one directed circuit that has $\Delta / M=2$, whereas $T_{s y s}=3$. This causes a warming-up period before the system goes to a periodic steady state. However, this is not the main issue, as most clocking systems have warming-up periods. The crucial point is that the MG does not satisfy (1) or (5) because the source is not the main controlling point and does not control the entire net. In other words, the constant delay shift in the periodic equation $\left(T_{s k e w_{n}}\right.$ in (1)) is not well defined. In Theorem 1, the SC property forced the $T_{\text {ske }} w_{n}$ value to be the delay of the zero tokens path from the source. However, when SC is violated as in the example of Figure 2, this skew value is instead determined by the critical circuit, which makes it not possible to express the firing of every transition using (1) with a well-defined notion of $T_{\text {skew }}$. 
We hereby substitute into (5) using the example to further elaborate on that point. The transitions firing should be written as follows:

$$
\begin{gathered}
\tau_{1}^{(i)}=\left(\max _{h \in \hat{\mathcal{H}}\left(t_{1}, t_{1}, 0\right)} \Delta(h)\right)+3 i=3 i \neq 1+3(i-1) \quad \forall i \geq 2 \\
\tau_{2}^{(i)}=\Delta\left(t_{1} p_{2} t_{2}\right)+3 i=1+3 i \neq 3 i \quad \forall i \geq 1 \\
\tau_{3}^{(i)}=\Delta\left(t_{1} p_{2} t_{2} p_{3} t_{3}\right)=2+3 i \quad \forall i \geq 0 \\
\tau_{4}^{(i)}=\Delta\left(t_{1} p_{2} t_{2} p_{3} t_{3} p_{6} t_{4}\right)=3+3 i \quad \forall i \geq 0 .
\end{gathered}
$$

We notice that the equations are wrong for $t_{1}$ and $t_{2}$ (the correct formula is written after the $\neq$ symbol). Both $t_{3}$ and $t_{4}$ are correct because they do belong to a critical circuit, and therefore the source has the power of initializing their first firing only, and after that, they control their own firings as in (44) (see Theorem 4).

\subsection{Practical Condition}

In this section, we assume a more practical case, where we cannot know the value of $\Delta_{M A X} a$ priori. Thus, the fourth constraint (see Section 4) is violated. We show that by only probing a single transition, such a system could be tuned to be used as an ACDN as discussed in Section 7. It is worth mentioning that this is the main motivation behind the work done in this article: the practical case of uncertainty in systems with a high level of variability.

\section{Theorem 2}

In an MG-LTS,

$$
\begin{gathered}
\text { if } C\left(t_{s}\right) \cap \arg \max _{C_{k} \in \mathcal{C}}\left(\frac{\Delta\left(C_{k}\right)}{M\left(C_{k}\right)}\right)=\emptyset, \\
\text { then } \exists i^{\star}, \exists t_{n} \in C_{n} \in C\left(t_{s}\right) \text {, and } \exists t_{u} \in \mathcal{T}, t_{u} \neq t_{s}, \\
\text { such that } \tau_{s}^{\left(i^{\star}\right)}+\max _{h \in \hat{\mathcal{H}}\left(t_{s}, t_{n}, 0\right)}(\Delta(h))<\tau_{u}^{\left(i^{\star}-M\left(h_{u \rightarrow n}\right)\right)}+\Delta\left(h_{u \rightarrow n}\right) .
\end{gathered}
$$

In other words, given an MG-LTS, if $t_{s}$ does not belong to a critical circuit (first line in the theorem), then there exists a certain occurrence $i^{\star}$ at which the value of the summation of the $i^{\star} t h$ firing of $t_{s}$ and the maximum token-less path delay between $t_{s}$ and $t_{n}$, where $t_{n}$ is a transition that shares a directed circuit with $t_{s}$, has to be less than the value of the summation of the $\left(i^{\star}-M\left(h_{u \rightarrow n}\right)\right) t h$ firing of $t_{u}$, where $t_{u}$ is a different transition from $t_{s}$, and the delay of a certain path from $t_{u}$ to $t_{n}-$ $h_{u \rightarrow n}$. The full definitions and nomenclature used are provided in Appendix A. The formal proof of the theorem is in Appendix B.4.

In simpler words, if an MG satisfies the conditions mentioned earlier, then there exists a period of time (i.e., after $i^{\star}$ occurrences), after which it is sufficient to probe the inputs of a single transition, $t_{n}$, to verify the condition that the source transition $t_{s}$ does not belong to a critical circuit, and thus fix this situation by increasing the delay of a circuit containing $t_{s}$. Otherwise, the critical circuit will be determined by some other transition $t_{u}$, which does not share a circuit with $t_{s}$.

It is worth mentioning that in case of stochastic delay values (see Definition 6 and Section 7), the preceding conditions will still hold, and the sequence of probing/increasing delay will force $t_{s}$ to eventually be in a critical circuit.

Clarifying example. In an attempt to clarify the intuition of Theorem 2, Figure 2 shows an MG example that violates the SC constraint as discussed in Section 5.1. Thus, $t_{n}$ in the theorem has to 
be $t_{2}$ because it is the only transition that shares a circuit with $t_{1}$, the source. And $t_{u}$ can be either $t_{3}$ or $t_{4}$. In case of $t_{3}$, for example, the least tokens path between $t_{3}$ and $t_{2}$ is the path $t_{3} p_{4} t_{2}$. And the longest zero tokens path between $t_{1}$ and $t_{2}$ is $t_{1} p_{2} t_{2}$. Following this example, we can substitute those into (7) as follows:

$$
\tau_{1}^{\left(i^{\star}\right)}+1<\tau_{3}^{\left(i^{\star}-1\right)}+1
$$

From the table with the transitions firing, we can see that the previous inequality is valid for $i^{\star} \geq 2$.

\section{COMPARISON TO PREVIOUS WORK}

This section situates our contribution within the relevant previous works and, as an example, highlights a specific hierarchical clocking structure that satisfies the ACDN structure in a technology with high levels of timing uncertainty.

\subsection{Circuits and Systems Literature}

First, any fully synchronous clocked system using an external clock source, such as a crystal oscillator in the case of PLL clocked systems, cannot be considered as an ACDN. Second, delay-insensitive circuits [7], including the ones that use dual-rail logic [17, 30], although they are indeed asynchronous, do not have any synchronous clock sinks because the logic is intrinsically embedded into the datapath. The property of (1) is not guaranteed, and they generally are not ACDNs. Third, systems that apply a GALS approach $[1,2,8,18,19]$, being either mesochronous or plesiochronous, and whether they use pausable clock interfaces, FIFO's interfaces, or typical synchronizers, all require one or more external clock inputs. Additionally, due to metastability issues, they cannot be modeled using deterministic delays [3]. Thus, they are also outside the scope of ACDNs.

We hereby mention a couple of systems that do fit into the ACDN category, with the first being the work of Cordatella et al. [5], where the authors proposed the use of an RO instead of a PLL. The clock being generated on-chip, they showed that the clock variations will be correlated to the datapath variations. Then they used a conventional clock tree to deliver the RO's output to the clock sinks. Regarding the second system [32, 33], it particularly benefits from every property of an ACDN, and that is the reason we choose to highlight it in this work. Figure 3 shows the heterogeneous clover-leaves clocking (HCLC) presented in Tadros and Beerel [31]. This design uses pairs of loops that only need a start-up signal and then continues to be periodic as determined by its slowest loop. Its application was focused on circular shift registers and the clock cycle time is $\mathrm{O}(\mathrm{N})$, where $\mathrm{N}$ is number of clocked elements; HCLC is an ACDN. In this work, however, we also highlight an evolved version of this design [31] whose clock cycle time is $\mathrm{O}(1)$ [33]. This clocking technique uses hierarchical chains of homogeneous clover leaves as asynchronous structures to provide the clocking signal for the very deep pipelines of single flux quantum (SFQ) circuits, a promising superconducting electronics technology [21]. This method allowed them to couple both the datapath and clock path to benefit from spatial correlation of most sources of variability leading to a more robust clocking scheme.

Figure 4 illustrates the MG model of a three-level hierarchy of the technique in Tadros and Beerel [32] using small numbers of links in every chain (two links in L2 and three links in L1). The red loop is the top loop containing the single source $t_{s}$ that is shaded; a programmable delay line should be inserted into this loop. The red transition is the transition that could be probed (see Section 7 and Theorem 2). The blue transitions could be considered as the synchronous clock sinks, although they are actually clovers of many gates similar to the structure in Tadros and Beerel [31]. This graph is an example of an MG that satisfies the design constraints of Section 4 and can thus be used as an ACDN. 


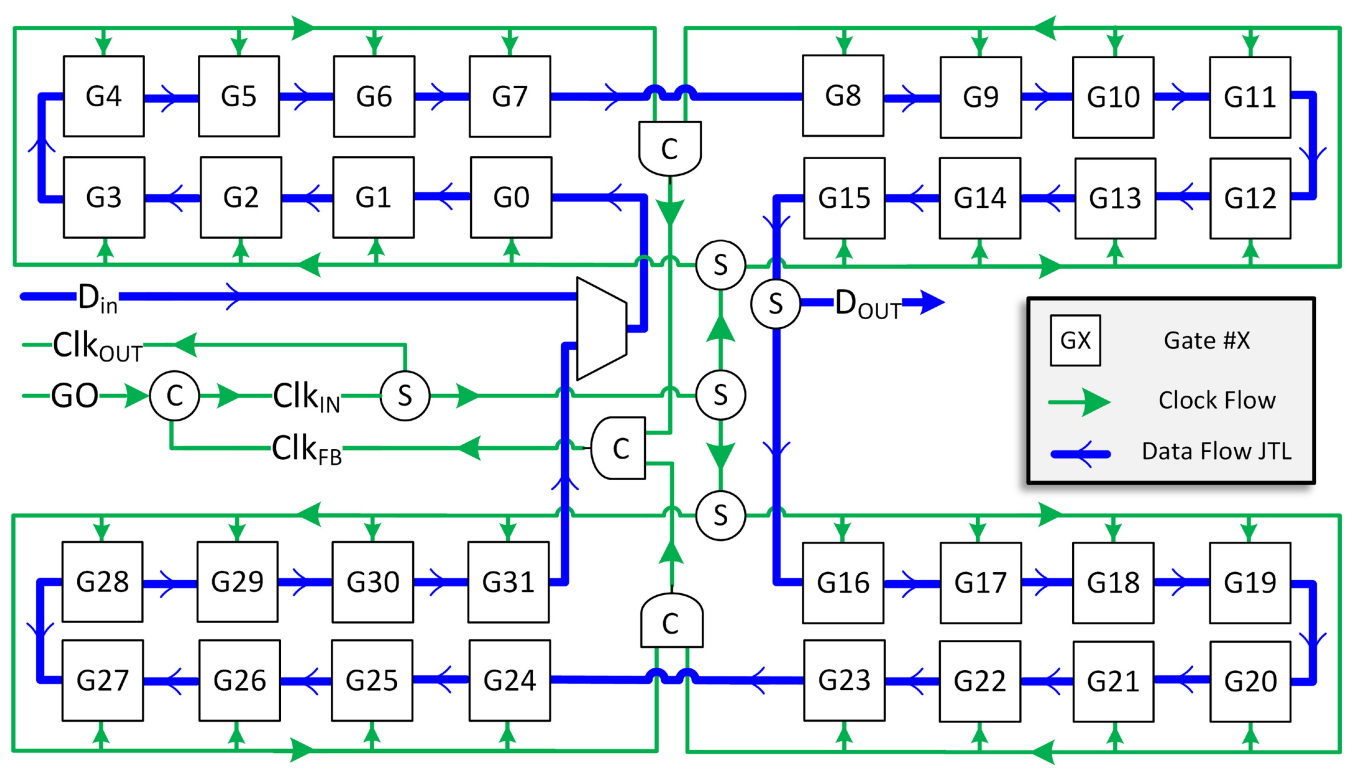

Fig. 3. The HCLC structure for a 32-gate circular shift register as designed by Tadros and Beerel [31].

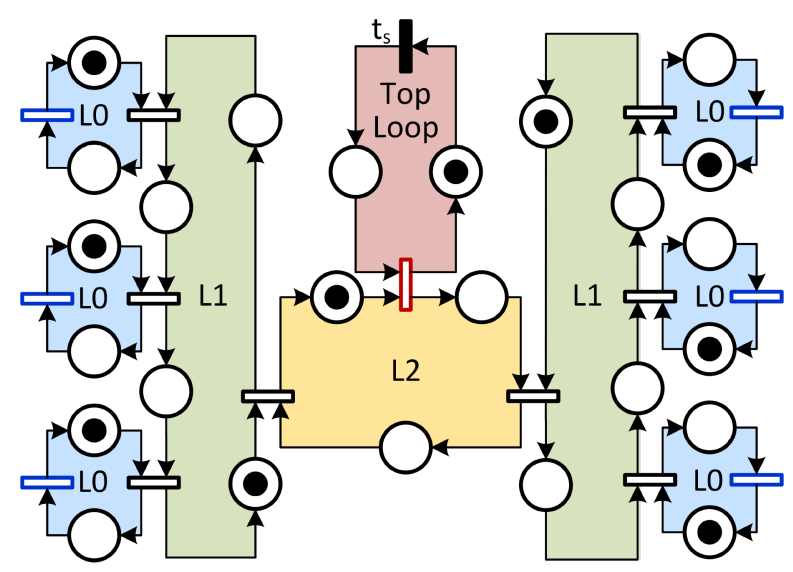

Fig. 4. The MG model of a simple hierarchy of the clocking technique in Tadros and Beerel [32]. LX stands for the Xth level.

It should also be noted that one of the famous concerns about asynchronous structures is the performance limitations, especially in the case of very short cycle times as in gate-level deep pipelines in SFQ [21,33]. When we suggest the use of asynchronous structures to provide the timing of a synchronous systems, a question emerges about the length of the longest loop that will be the frequency bottleneck. In the work of Tadros and Beerel [31], for example, the cycle time is a function of the number of clock sinks. This renders such an ACDN design impractical to be used for large synchronous systems. This is a significant advantage of the work of Tadros and Beerel [32, 33], whose cycle time is independent of the number of clock sinks. We state that it is essential for an ACDN to have performance that is $\mathrm{O}(1)$ w.r.t. clock sinks. 
In this article, the concept of ACDN that we formalize and introduce targets a different compromise of both performance and robustness, especially in the applications where high timing uncertainty is a concern as designed in Theorem 2. We suggest the elimination of a clock source and clock tree in the conventional sense, and replace it with an asynchronous structure that selfadapts to such uncertainty and endorse the coupling of data- and clock paths to benefit from its intrinsic timing robustness [11].

\subsection{Theoretical Literature}

First, Magott [22] and Ramamoorthy and Ho [27] have reached similar results to Theorem 1 in this article. However, they have not used the same conditions that we specified. We henceforth show that they have under-constrained their theorems. In particular, both Theorem 3 of Ramamoorthy and Ho [27] and Theorem 1 of Magott [22] state that transitions of timed MGs satisfy

$$
\tau_{n}^{(i)}=\tau_{n}^{(0)}+i \cdot T_{\text {sys }}, \quad \forall i \geq 0,
$$

which is identical to the results of our Theorem 1 but without the theorem's constraints. Figure 1 and Figure 2 shows two counter-examples that support our claim. The tables next to the diagrams shows the first six transitions firings. Figure 1 shows an MG that violates both the single source and the single token per directed circuit constraints. Similar to the discussion in Section 4, the period of the transitions firings of the MG in Figure 1 oscillates, and therefore their behavior cannot be described using (9). Regarding the example in Figure 2, which is discussed in Section 5.2, that MG indeed has a single token per circuit and a single source. However, the source does not belong to the critical circuit. This causes the following: first, a warming-up period before the system goes to a periodic steady state that already violates (9), and second, a constant delay shift that varies from one transition to another based on how they are connected to the critical circuit. This is discussed in Section 5.2, and it is more complicated than described using the simple theorem of (9).

The works of Hua and Manohar [13] and Hulgaard et al. [15] are also tightly related to this article. The work of Hulgaard et al. [15] modeled concurrent systems using a process graph where the vertices represent the events and the edges represent the rule templates (i.e., the events dependency and relations). To describe the events periodicity and repetition, they used an occurrence index (similar to $(i)$ in this article), and they did unfolding of the process graph to obtain an infinite directed graph that describes every iteration in an explicit manner. They used the concept of an execution model (see Appendix B.1) to represent the consistent assignment of time values to event occurrences. The main contribution of the work of Hulgaard et al. [15] was to develop an algorithm that can be used to find the maximum time separation between two events in a finite portion of the unfolded process graph-that is, given that each event is bounded by a minimum delay value $\delta$ and a maximum one $\Delta$.

For the sake of this article's scope, we recite the following conclusion from the work of Hulgaard

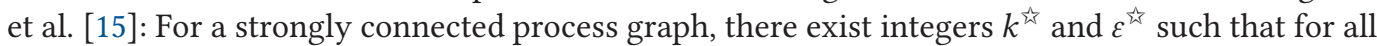
$k \geq k^{\overparen{k}}+\beta$, and any event (vertex) $v$ in the graph,

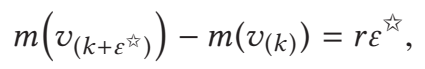

where $m\left(v_{(k)}\right)$ is the longest time path (using the lower bounds $\delta$ ) between the $k t h$ occurrence of the event $v$ and the $\beta$ th occurrence of another event $u$, and $r$ is the maximum ratio cycle (the counterpart of $\triangle_{M A X}$ in this article). They defined $k^{\text {th }}$ as the number of unfoldings of the process graph backward relative to the $\beta$ th occurrence of the event $u$, and $\varepsilon^{\text {th }}$ as the occurrence period of this repetition. Those integers, $k^{\not{t}}$ and $\varepsilon^{\vec{k}}$, are values specific to a particular process graph.

In simpler words, this means that after a certain warming-up period or an uncertainty period, which happens also after a certain number of occurrences, the TSE between two events shall reach 
a periodic steady state. It is worth mentioning that for an MG-LTSC (see Theorem 1), our results prove that those integers, $k^{\widehat{t}}$ and $\varepsilon^{\vec{k}}$, are equal to zero and one, respectively.

The work of Hua and Manohar [13] modeled the systems using an event rule (ER) paradigm, where $E$ is a set of events and $R$ is a set of rules that defines the dependency and the timing constraints. They defined a repetitive event rule (RER) system where the events do repeat, and thus they defined an occurrence index $i$ (similar to this article), and they defined an occurrence index offset $\epsilon$ that they associate with a rule. This is the counterpart of the number of markings (tokens) that separate two transitions in an MG. The main contribution of the work of Hua and Manohar [13] was to develop a set of algorithms and rules that can be used to compute the exact timing behavior of more general classes of systems that combine asynchronous and synchronous structures.

For the sake of this article, we recite the following conclusion from the work of Hua and Manohar [13]: In any RER system (note that an MG-L is an RER system by definition), there exists an integer $M$ and a bound $k^{\star}$ such that the system's periodicity can be described for any event $e$, for $n \geq k^{\star}$, as follows:

$$
\hat{t}(\langle e, n+M\rangle)-\hat{t}(\langle e, n\rangle)=M p^{\star},
$$

where $\hat{t}(\langle e, n\rangle)$ is the exact time of the $n t h$ occurrence of the event $e, p^{\star}$ is the maximum cycle (the counterpart of $\triangle_{M A X}$ in this article), and $M$ is the system's periodicity.

This is similar to the conclusion reached by the work of Hulgaard et al. [15] in the previous section. It is worth mentioning that for an MG-LTSC (see Theorem 1), our results prove that those integers, $k^{\star}$ and $M$, are equal to zero and one, respectively.

As mentioned in Section 3, a periodic MG-L has a warm-up period before the system enters a periodic steady state $[13,15]$. This can be formalized in the existence of integers $m^{\star}$ and $i^{\star}$ that satisfy the following:

$$
\tau_{u}^{\left(i+m^{\star}\right)}-\tau_{u}^{(i)}=m^{\star} \cdot \Delta_{M A X}, \forall i \geq i^{\star}, \forall t_{u} \in \mathcal{T},
$$

where $m^{\star}$ is the occurrence period of repetition (i.e., the system periodicity) and $i^{\star}$ is the occurrence warming-up bound before the periodic steady state. This formalization is similar to the ones introduced by Hua and Manohar [13] and Hulgaard et al. [15]. It should be noted that both $m^{\star}$ and $i^{\star}$ are characteristic to a graph rather than to a specific transition.

If we applied the formulations of Appendix B.2 in Hulgaard et al. [14] or algorithm-1 in Hua and Manohar [13] to an MG-LT, both would lead to the same conclusion of $m^{\star}=1$, which supports our conclusions in Theorem 1. In particular, if we apply this to $t_{s}$ under the conditions of Theorem 2, we can write

$$
\tau_{s}^{(i+1)}-\tau_{s}^{(i)}=\Delta_{M A X}, \quad \forall i \geq i^{\star} .
$$

This means that (7) is applicable for any $i \geq i^{\star}$.

The authors of Hua and Manohar [13] have determined the value of the upper bound of $i^{\star}$ in the worst-case scenario (see Theorem 3 in Hua and Manohar [13]). For an MG-LT and $m^{\star}=1$, their upper bound becomes

$$
i^{\star} \leq\left(4+\frac{3 \cdot \Delta_{M A X}}{\Delta_{M A X}-\Delta_{2^{n d}} M A X}\right) \cdot|\mathcal{P}|,
$$

where $\Delta_{2^{n d}}$ MAX is the second largest circuit (see Lemma 3). In contrast, this work shows that for MG-LTSC systems, $i^{\star}=0$, and $\tau_{n}^{(0)}$ is well determined for any transition $t_{n}$. We provide two examples to clarify this point:

(1) The graph of Figure 1 is an example for a net with a system periodicity larger than one. In particular, $m^{\star}=2$ for that $M G-$ that is, the transitions firing of the net in Figure 1 can 
be described following (12) as follows:

$$
\tau_{u}^{(i+2)}-\tau_{u}^{(i)}=2 \Delta_{M A X}, \forall i \geq 0, \forall t_{u} \in \mathcal{T} .
$$

(2) Following the discussion in Section 5.2 about the example of Figure 2, we can see that $\Delta_{M A X}=3, \Delta_{2^{n d}}$ MAX $=2$, and $|\mathcal{P}|=6$. Therefore, we substitute into (14) to get the limit of $i^{\star}$ as follows:

$$
i^{\star} \leq\left(4+\frac{3 \times 3}{3-2}\right) \times 6=78
$$

It is noticeable that this is a conservative upper bound since following the transitions firings we can deduce that $i^{\star}=2$.

Another work that needs to be mentioned is the work of Nielsen and Kishinevsky [26], in which the authors focused on efficient means of computing performance of signal graphs that are an extension of MGs. They used unfoldings of the graph and timing simulations from a cut set of the graph to analyze its performance. Like the preceding techniques, they do not identify a sub-class of MGs for which the analysis can be derived from a single graph node.

Regarding the work of McGee and Nowick [23], which has the closest ties to our work, the authors also focused on designing an algorithm that finds the TSE of a given graph. In addition to that, they identified a sub-class of live and safe MGs that has a single token per directed circuit, a single critical circuit, and a single source that belongs to that critical circuit. They reached similar conclusions to our Theorem 1 . However, as our derivations to Theorem 1 show, there is no need to over-constrain the conditions with a single critical circuit as long as the single source belongs to one of the critical circuits of the graph. Moreover, our work provides formal and complete proofs that lead to the conclusions of McGee and Nowick [23]. Furthermore, and the most crucial point when discussing ACDNs, our work studies the case when it is not guaranteed for the source to be in a critical circuit. Such practical uncertainty is the main motivation behind this work.

\section{DISCUSSION}

When we compare Theorem 1 and Corollary 4 to (1), we find that every transition does indeed fire periodically every $T_{s y s}$ with no warming-up phase (both $T_{u p}$ and $i_{u p}$ equal zero). Regarding $T_{\text {skew }}$, it is well defined and equals the initial delay between the source and $t_{n}$. This means that if the MG model of an asynchronous system is an MG-LTSC, it can be used as an ACDN for a synchronous system.

The four constraints of Section 4 can be interpreted as follows. First, the absence of an alwaysenabled-transition [25] excludes systems with externally generated clock sources. Second, a live and safe MG guarantees the firing periodicity. Third, only one token per directed circuit guarantees a fixed clock period (i.e., $\Gamma_{n}=T_{\text {sys }}$ and $m^{\star}=1$. 4). A single source that belongs to a critical circuit guarantees the a priori knowledge of $T_{u p}$ and the clock skew $T_{s k e w_{n}}$ that provides the needed timing information for a robust CDN against setup and hold violations [34]. Those constraints can thus be considered as design guidelines for an asynchronous system to be used as a CDN for a synchronous system.

Nevertheless, as shown in Section 5.2, if the fourth constraint is partially satisfied (only MGLTS), then there exists a transition that shares a circuit with the source such that probing it enables finding the value of $T_{\text {sys }}$. This is one of the foundations of the practicality of the clocking structurehighlighted in this document. By probing the inputs of this transition, a programmable delay line inserted into one of the circuits with $t_{s}$ can be adjusted until the system becomes an MG-LTSC. Henceforth, the system will behave as an ACDN.

An important point that is worth mentioning is our choice of using deterministic delay values for the sake of simplicity. Practically, and particularly in the case of uncertainties, all delay values 


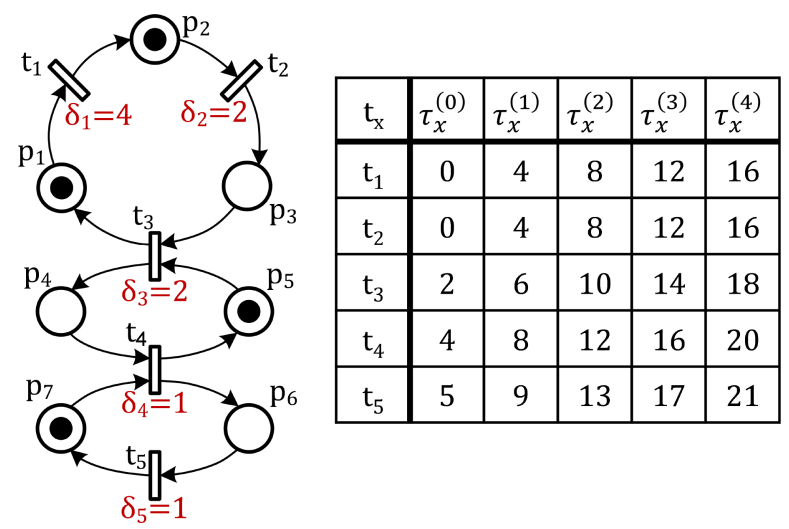

Fig. 5. An example of an MG that violates the sufficient constraints but still satisfy (1) and (5). $T_{\text {sys }}=$ $\max _{C \in C}\left(\frac{\Delta(C)}{M(C)}\right)=\frac{8}{2}=4$.

would possess a stochastic value rather than a deterministic one because of variations, or design asymmetries, and so forth. In such a case, every delay value shall be presented by a range with a lower and an upper bound (similar to the model in Hulgaard et al. [15]). Under such a model, it will be possible to study time-varying clock skews and timing jitter.

A final point that needs to be discussed is that throughout this article, we emphasize that we are providing sufficient conditions to satisfy (1). We hereby discuss the necessary conditions. First, we show an example that violates the constraints and still satisfy (1) to prove that our conditions are not necessary. In the example of Figure 5, the net violates both the $\mathrm{T}$ and the $\mathrm{S}$ constraints-that is, not every circuit has a single token, and there is more than one source. However, as we can see from the table of the transition firings, all transitions can be written following (1) or Theorem 1 considering $t_{1}$ as the source for $t_{1}$ and $t_{2}$ as the source for the rest. We write the equations for $\forall i \geq 0$ to elaborate

$$
\begin{gathered}
\tau_{1}^{(i)}=\left(\max _{h \in \hat{\mathcal{H}}\left(t_{1}, t_{1}, 0\right)} \Delta(h)\right)+4 i=4 i \\
\tau_{2}^{(i)}=\left(\max _{h \in \hat{\mathcal{H}}\left(t_{2}, t_{2}, 0\right)} \Delta(h)\right)+4 i=4 i \\
\tau_{3}^{(i)}=\Delta\left(t_{2} p_{3} t_{3}\right)+4 i=2+4 i \\
\tau_{4}^{(i)}=\Delta\left(t_{2} p_{3} t_{3} p_{4} t_{4}\right)+4 i=4+4 i \\
\tau_{5}^{(i)}=\Delta\left(t_{2} p_{3} t_{3} p_{4} t_{4} p_{6} t_{5}\right)+4 i=5+4 i .
\end{gathered}
$$

However, the example of Figure 1 violates the same two constraints but causes oscillation in the cycle time as discussed in Section 4. That is why we believe that one of the necessary conditions is that every path $h$ from any transition to a source has to satisfy $\Delta(h)-T_{s y s} \cdot M(h) \leq 0$. This inequality is inspired from the lemma that the work of Ramamoorthy and Ho [27] used to prove their Theorem 3. It has to be mentioned that they proved this inequality for every circuit, but they incorrectly applied it to every path, which led to an ill-constrained theorem as we showed in Section 6.2. In particular, it is trivial to verify that this inequality is violated by the path $\left\{t_{1} p_{2} t_{2} p_{3} t_{3}\right\}$ in the MG depicted in Figure 1. The key intuition behind our result is that by forcing every directed circuit to have one token and by having a single source, then this inequality is indeed satisfied for all paths in the MG. 
In summary, we believe that the necessary conditions to satisfy Theorem 1 are too complex to be useful for designing ACDNs. However, by introducing more conservative conditions, which are sufficient, we simplify the design requirements and make it practical for circuit designers to verify whether their asynchronous structures can indeed be used to provide the clocking signals for a synchronous system.

\section{CONCLUSION}

This article provides the theoretical foundation for ACDNs. Particularly, we show sufficient constraints for a system with asynchronous structures to provide strictly and well-determined periodic signals for a large number of clock sinks, which is necessary for the timing of a fully synchronous system. We use timed MGs to prove that for a live and safe MG with a single token per directed circuit, if there exists a single source, and this source belongs to one of the critical directed circuits, then all transitions (or clock sinks) will fire periodically and with well-determined clock time occurrences. This allows the timing of all clock sinks in a synchronous manner in the absence of a clock source. Additionally, we prove that by only probing a single transition and adjusting a single programmable delay line, we are able to force the source to belong to a critical circuit, thus forcing a well defined synchronous periodicity. This is particularly useful in systems with high levels of uncertainty.

\section{APPENDICES}

\section{A DEFINITIONS AND NOMENCLATURE}

\section{A.1 Basic Definitions}

For the sake of completeness and nomenclature consistency, we start this section with some basic definitions about timed Petri nets (PNs) and marked graph (MG) theory [25]. It should be noted that the work in this article exclusively considers circuits modeled as MGs.

Definition 6 (Petri Net). The Petri net $(\mathrm{PN})$ in our manuscript is a 4-tuple, $P N=\left(\mathcal{P}, \mathcal{T}, \mathcal{F}, \mathcal{M}_{0}\right)$ [25], where $\mathcal{P}$ is the finite set of places, $\mathcal{T}$ is the finite set of transitions, $\mathcal{F} \subset(\mathcal{P} \times \mathcal{T}) \cup(\mathcal{T} \times \mathcal{P})$ is the finite set of arcs describing the flow relation, and $\mathcal{M}_{0}$ is the initial marking.

In this article, we study timed $\mathrm{PN}$; we choose to associate time delays with transitions. For every $t_{n} \in \mathcal{T}, \delta_{n} \geq 0$ is used to represent this value. It is worth mentioning that we primarily focus on a deterministic PN where all delay values are deterministically defined-that is, not stochastic, but we consider extensions in Section 7.

Definition 7 (Marked Graph). A marked graph (MG) is a PN such that each place $p$ has exactly one input transition and exactly one output transition [25]. Figure 6 shows an example of a timed MG. Note that a live and safe MG (see Definitions 12 and 13) is a strongly connected graph by definition.

Definition 8 (Places). $p_{n} \in \mathcal{P}$ denotes the $n t h$ place, and $m_{n} \in \mathcal{M}_{0}$ is the initial number of markings in $p_{n}$. In Figure $6, p_{1}, p_{3}$, and $p_{8}$ are examples of marked places (i.e., $m_{1}=m_{3}=m_{8}=1$ ), whereas the rest of places are unmarked and thus have $m=0$.

Definition 9 (Transitions). $t_{n} \in \mathcal{T}$ denotes the $n t h$ transition, and $\delta_{n}$ is the execution time or the delay associated with $t_{n}$. In Figure 6, the delays are shown in red below the associated transitions.

Definition 10 (Firing Rule). A transition $t_{n}$ is enabled if each input place $p_{\text {in }}$ of $t_{n}$, where $p_{\text {in }} \in$ $\mathcal{P} \wedge p_{\text {in }} t_{n} \in \mathcal{F}$, has a token. After $\delta_{n}$, one token is removed from each input $p_{i n}$, and one token is added to every output place $p_{\text {out }}$, where $p_{\text {out }} \in \mathcal{P} \wedge t_{n} p_{\text {out }} \in \mathcal{F}$ [25]. For example, in Figure 6, 


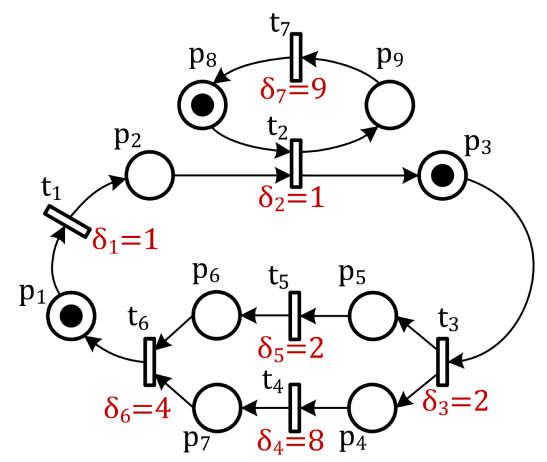

Fig. 6. An example of a timed MG.

$t_{3}$ has one input place, $p_{3}$, and it has an initial marking. Therefore, at $t=0, t_{3}$ initiates the firing and consumes the marking of $p_{3}$. Then, at $t=2, t_{3}$ fires since $\delta_{3}=2$, and both $p_{4}$ and $p_{5}$ become marked with one marking each. After that, both $t_{4}$ and $t_{5}$ initiate their firing and fire at $t=10$ and $t=4$, respectively.

Definition 11 (Transition Firing). $\tau_{n}^{(i)}$ is the time at which transition $t_{n}$ initiates its ith firing. Following the same example of Figure $6, \tau_{3}^{(0)}=0, \tau_{4}^{(0)}=\tau_{5}^{(0)}=2$.

Definition 12 (Liveness). A PN is live if it is always possible to ultimately fire any transition guaranteeing a deadlock-free operation [25]. Formally, a PN is live if $\tau_{n}^{(i)}$ is defined for $\forall i \geq 0, \forall t_{n} \in$ $\mathcal{T}$.

Definition 13 (Safeness). A PN is safe if the number of tokens in each place does not exceed one [25].

Definition 14 (Sources). The sources set $\mathcal{S}$ is defined such that a transition $t_{s} \in \mathcal{S}$ if it is enabled initially (these transitions are called roots in Hulgaard et al. [15]). Note that $\mathcal{S} \subset \mathcal{T}$, and we set $\tau_{s}^{(0)}=0$ for all $t_{s} \in S$. In Figure $6, t_{1}$ and $t_{3}$ are the only sources.

\section{A.2 Paths}

In this section, we include some established definitions regarding paths, in addition to presenting the useful concepts of token-limited directed path set and directed path length.

Definition 15 (Directed Path). A directed path [24] is a sequence of alternating transitions and places. $t_{1} p_{1} t_{2} p_{2} \cdots t_{N}$ is a directed path from $t_{1}$ to $t_{N}$ if $t_{n} p_{n} \in \mathcal{F}$ and $p_{n} t_{n+1} \in \mathcal{F}$ for $1 \leq n \leq N-1$ $[24,27]$. We use the symbol " $\sqsubset$ " as in $h_{1} \sqsubset h_{2}$ to denote that the path $h_{1}$ is a section or a subsequence of the path $h_{2}$. For example, in Figure 6, let us denote the path $t_{2} p_{3} t_{3}$ as $h_{1}$ and the path $t_{1} p_{2} t_{2} p_{9} t_{7} p_{8} t_{2} p_{3} t_{3}$ as $h_{2}$. Thus, $h_{1} \sqsubset h_{2}$.

Definition 16 (Directed Path Delay). We define the delay of a directed path as the sum of the execution times of the transitions forming the path $h$ excluding the target transition-that is, it is the delay from the enabling of the start transition until the enabling of the end transition. This can be formalized as follows for a path $h$ from $t_{\text {start }}$ to $t_{\text {end }}$ :

$$
\Delta(h)=\left(\sum_{t_{j} \in h} \delta_{j}\right)-\delta_{\text {end }}
$$

In Figure 6, $\Delta\left(h_{1}\right)=1$ and $\Delta\left(h_{2}\right)=12$. 
Definition 17 (Directed Path Set). We define $\mathcal{H}\left(t_{u}, t_{v}\right)$ as the set of all possible directed paths from $t_{u}$ to $t_{v}$. This set can be infinite for an MG due to the presence of loops. For example, in Figure $6, \mathcal{H}\left(t_{2}, t_{7}\right)=\left\{t_{2} p_{9} t_{7}, t_{2} p_{9} t_{7} p_{8} t_{2} p_{9} t_{7}, \ldots, t_{2} p_{3} t_{3} p_{5} t_{5} p_{6} t_{6} p_{1} t_{1} p_{2} t_{2} p_{9} t_{7}, \ldots\right.$, $\left.t_{2} p_{3} t_{3} p_{4} t_{4} p_{7} t_{6} p_{1} t_{1} p_{2} t_{2} p_{9} t_{7} p_{8} t_{2} p_{9} t_{7}, \ldots\right\}$.

Definition 18 (Directed Path Synchronic Distance). In a similar concept to the synchronic distance between two transitions in Leu [20], we define the synchronic distance of a directed path, $h \in$ $\mathcal{H}\left(t_{u}, t_{v}\right)$, from $t_{u}$ to $t_{v}$ as the number of times that one transition can fire without firing the other according to that path. Formally, we write it as

$$
M(h)=\sum_{p_{j} \in h} m_{j}
$$

In Figure 6, $M\left(h_{1}\right)=1$ and $M\left(h_{2}\right)=2$.

Definition 19 (Token-Limited Directed Path Set). We define $\hat{\mathcal{H}}\left(t_{u}, t_{v}, m\right)$ as the set of all directed paths from a transition $t_{u}$ to a transition $t_{v}$ that have a directed path synchronic distance less than or equal to the integer $m$. This can be formalized as follows:

$$
\hat{\mathcal{H}}\left(t_{u}, t_{v}, m\right)=\left\{h: h \in \mathcal{H}\left(t_{u}, t_{v}\right) \wedge M(h) \leq m\right\} .
$$

Using the same example as before, $\hat{\mathcal{H}}\left(t_{2}, t_{7}, 0\right)=\left\{t_{2} p_{9} t_{7}\right\}, \hat{\mathcal{H}}\left(t_{2}, t_{7}, 1\right)=\left\{t_{2} p_{9} t_{7}, t_{2} p_{9} t_{7} p_{8} t_{2} p_{9} t_{7}\right\}$, and $\hat{\mathcal{H}}\left(t_{2}, t_{7}, 2\right)=\left\{t_{2} p_{9} t_{7}, \quad t_{2} p_{9} t_{7} p_{8} t_{2} p_{9} t_{7}, \quad t_{2} p_{9} t_{7} p_{8} t_{2} p_{9} t_{7} p_{8} t_{2} p_{9} t_{7}, \quad t_{2} p_{3} t_{3} p_{5} t_{5} p_{6} t_{6} p_{1} t_{1} p_{2} t_{2} p_{9} t_{7}\right.$, $\left.t_{2} p_{3} t_{3} p_{4} t_{4} p_{7} t_{6} p_{1} t_{1} p_{2} t_{2} p_{9} t_{7}\right\}$.

Definition 20 (Directed Path Length). We define the length of a directed path $h \in \mathcal{H}\left(t_{u}, t_{v}\right)$ as the number of transitions in that path excluding the end transition, $t_{v}$. We denote the path length as $|h|$. We also use $\mathcal{H}_{l}\left(t_{u}, t_{v}\right)$ to denote the set of all possible directed paths from $t_{u}$ to $t_{v}$ with length $\leq l$. Formally, it can be written as follows:

$$
\mathcal{H}_{l}\left(t_{u}, t_{v}\right) \subset \mathcal{H}\left(t_{u}, t_{v}\right) ; \mathcal{H}_{l}=\left\{h: h \in \mathcal{H}\left(t_{u}, t_{v}\right) \wedge|h| \leq l\right\} .
$$

Such a set can also be further constrained with a token bound as follows:

$$
\hat{\mathcal{H}}_{l}\left(t_{u}, t_{v}, m\right)=\left\{h: h \in \mathcal{H}_{l}\left(t_{u}, t_{v}\right) \wedge M(h) \leq m\right\} .
$$

Using the same example, $\mathcal{H}_{1}\left(t_{2}, t_{7}\right)=\left\{t_{2} p_{9} t_{7}\right\}, \quad \mathcal{H}_{3}\left(t_{2}, t_{7}\right)=\left\{t_{2} p_{9} t_{7}, \quad t_{2} p_{9} t_{7} p_{8} t_{2} p_{9} t_{7}\right\}, \quad$ and $\mathcal{H}_{3}\left(t_{2}, t_{7}, 0\right)=\left\{t_{2} p_{9} t_{7}\right\}$.

Definition 21 (Loop). A directed path $t_{1} p_{1} t_{2} p_{2} \cdots t_{N}$ is a loop if $t_{1}=t_{N}$. From Figure 6 , the following paths are loops: $t_{2} p_{9} t_{7} p_{8} t_{2}, t_{2} p_{9} t_{7} p_{8} t_{2} p_{9} t_{7} p_{8} t_{2}, t_{1} p_{2} t_{2} p_{3} t_{3} p_{5} t_{5} p_{6} t_{6} p_{1} t_{1}$, and $t_{2} p_{9} t_{7} p_{8} t_{2} p_{3} t_{3} p_{4} t_{4} p_{7} t_{6} p_{1} t_{1} p_{2} t_{2}$.

Definition 22 (Simple Directed Path). A simple directed path is a directed path from $t_{1}$ to $t_{N}$ where all transitions are distinct $[24,27]$. From Figure 6, the path $t_{2} p_{9} t_{7}$ is a simple directed path between $t_{2}$ and $t_{7}$, whereas the path $t_{2} p_{9} t_{7} p_{8} t_{2} p_{9} t_{7}$ is not.

\section{A.3 Performance}

In this section, we include some established definitions regarding circuits and cycle time [13, 15, $22,25,27,28]$, in addition to presenting the concept of firing period, which conveys the intuition of a strictly periodic clock signal in synchronous systems (see Section 3).

Definition 23 (Directed Circuit). A sequence $t_{1} p_{1} t_{2} p_{2} \cdots t_{N}$ is a directed circuit, $C$, if the path from $t_{1}$ to $p_{N-1}$ is a simple directed path, $p_{N-1} t_{1} \in \mathcal{F}$ and $t_{1}=t_{N}$ [22]. We let the set $C$ denote the set of all directed circuits in the PN. Two directed circuits that contain the same set of places and transitions are considered equivalent. We use $M\left(C_{k}\right)$ to denote the total number of tokens in the 
places of the $k t h$ directed circuit, $C_{k}$, and $\Delta\left(C_{k}\right)$ to denote the sum of the execution times of the transitions forming $C_{k}$. We also use $C\left(t_{n}\right)$ to denote the set of circuits containing the transition $t_{n}$. These can be formalized as follows for a circuit $C_{k}$ that starts and ends at transition $t_{u}$ :

$$
\begin{gathered}
M\left(C_{k}\right)=\sum_{p_{j} \in C_{k}} m_{j}, C_{k} \in C \\
\Delta\left(C_{k}\right)=\left(\sum_{t_{j} \in C_{k}} \delta_{j}\right)-\delta_{u}, C_{k} \in C \\
C\left(t_{n}\right)=\left\{C: C \in C \wedge t_{n} \in C\right\} .
\end{gathered}
$$

From Figure 6, the set $C=\left\{C_{1}, C_{2}, C_{3}\right\}$, where $C_{1}=\left\{t_{2} p_{9} t_{7} p_{8} t_{2}\right\}, C_{2}=\left\{t_{1} p_{2} t_{2} p_{3} t_{3} p_{5} t_{5} p_{6} t_{6} p_{1} t_{1}\right\}$, and $C_{3}=\left\{t_{1} p_{2} t_{2} p_{3} t_{3} p_{4} t_{4} p_{7} t_{6} p_{1} t_{1}\right\}$. For those circuits, $M\left(C_{1}\right)=1, M\left(C_{2}\right)=M\left(C_{3}\right)=2, \Delta\left(C_{1}\right)=10$, $\Delta\left(C_{2}\right)=10$, and $\Delta\left(C_{3}\right)=16$. In addition, $C\left(t_{1}\right)=\left\{C_{2}, C_{3}\right\}, C\left(t_{2}\right)=C, C\left(t_{7}\right)=\left\{C_{1}\right\}$.

Definition 24 (PN Cycle Time). A PN has a PN cycle time that is defined in Murata [25] as the time to complete a firing sequence leading back to the starting marking after firing each transition at least once.

There is a crucial distinction that must be made here since the term cycle time is used differently across many works [13, 15, 22, 25, 27, 28]. We use the complete term PN cycle time as a property related to the initial marking and the system periodicity [13] as defined previously, whereas we use cycle time for the other definition, which is oriented toward transition firing periodicity.

Definition 25 (Cycle Time). We define the cycle time as the mean time to complete a firing sequence leading to the firing of each transition for exactly once. It can be formalized as follows [22]:

$$
\hat{T}_{n}=\lim _{i \rightarrow \infty} \frac{\tau_{n}^{(i)}}{i}, \forall t_{n} \in \mathcal{T} .
$$

Definition 26 (Firing Period). We define the firing period of a transition as the exact cycle timethat is, the time separation between the $i$ th transition firing and the $(i+1)$ th firing:

$$
\Gamma_{n}^{(i)}=\tau_{n}^{(i+1)}-\tau_{n}^{(i)}, \forall t_{n} \in \mathcal{T}, \forall i \geq 0
$$

\section{B ACDN PROOFS}

In this appendix, we provide the mathematical derivations that prove the theoretical foundation for ACDNs modeled as MGs. In particular, we present the key lemmas and theorems with their proofs.

\section{B.1 Execution Model}

In this section, we apply the firing rule to formalize the description of any transition firing. In other words, for any transition $t_{n}$, we introduce an equation to formalize any transition occurrence $\tau_{n}^{(i)}$ for any arbitrary PN.

Definition 27 (Execution Model). In a PN, $\forall t_{v} \in \mathcal{T}, \forall i \geq 0$

$$
\tau_{v}^{(i)}=\max _{t_{u} \in \mathcal{T}}\left(\max _{h \in \hat{\mathcal{H}}_{1}\left(t_{u}, t_{v}, i\right)}\left(\tau_{u}^{(i-M(h))}+\delta_{u}\right)\right) .
$$

As in Hulgaard et al. [15], we define the execution of the $\mathrm{PN}$ as the consistent assignment of time values to the transitions firings. Using our previous definitions, Equation (26) is a direct translation of Equation (1) in Hulgaard et al. [15], which is a direct application to the firing rule of PNs [25]. This formalization follows the same conceptual understanding of the process graph unfolding 
[15] and the unrolling of repetitive event rule systems [13] (see Section 6.2). In simpler terms, Equation (26) means that the time of execution of the ith occurrence of the transition $t_{v}$ depends on the firing of the transitions that have one place separating them from $t_{v}$. In other words, the ith occurrence of $t_{v}$ has to come after the $(i-M(h)) t h$ occurrence of the latest transition to fire among those transitions. Since the path $h$ here is limited to be of unity length, $M(h)$ shall equal either zero or one depending on the initial marking of the place that separates $t_{u}$ and $t_{v}$ in $h$.

\section{Theorem 3}

In an $\mathrm{PN}, \forall t_{v} \in \mathcal{T}, \forall i \geq 0$

$$
\tau_{v}^{(i)}=\max _{t_{u} \in \mathcal{T}}\left(\max _{h \in \hat{\mathcal{H}}\left(t_{u}, t_{v}, i\right)}\left(\tau_{u}^{(i-M(h))}+\Delta(h)\right)\right) .
$$

In other words, the execution model can be generalized for any path with unlimited number of tokens.

Proof. From the execution model definition in (26), we use induction to extend this definition from the paths within a path length of one to the set of paths $\hat{\mathcal{H}}$ with unbounded length:

(a) The basis step is the definition of (26) itself, for a maximum length $l=1$.

(b) Let us assume that the following is true, for a maximum length $L, \forall t_{v} \in \mathcal{T}, \forall i \geq 0$

$$
\tau_{v}^{(i)}=\max _{t_{u} \in \mathcal{T}}\left(\max _{h \in \hat{\mathcal{H}}_{L}\left(t_{u}, t_{v}, i\right)}\left(\tau_{u}^{(i-M(h))}+\Delta(h)\right)\right) .
$$

(c) Let the arg max of those maximum sets (i.e., the transition at the beginning of the critical path, and the critical path itself) be $t_{x}$ and $h_{x}$, respectively. Applying the basis step to the transition $t_{x}$, we get $\forall i \geq 0$

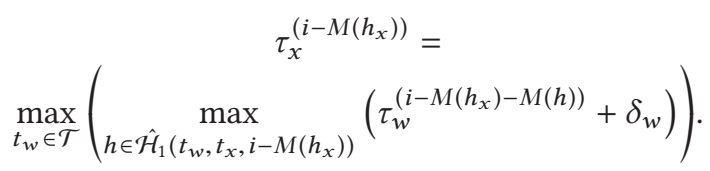

(d) Combining both (28) and (29), since $\hat{\mathcal{H}}_{1}\left(t_{w}, t_{x}, i-M\left(h_{x}\right)\right) \cup \hat{\mathcal{H}}_{L}\left(t_{x}, t_{v}, i\right)=\hat{\mathcal{H}}_{L+1}\left(t_{w}, t_{v}, i\right)$ by definition, we directly get $\forall t_{v} \in \mathcal{T}, \forall i \geq 0$

$$
\tau_{v}^{(i)}=\max _{t_{w} \in \mathcal{T}}\left(\max _{h \in \hat{\mathcal{H}}_{L+1}\left(t_{w}, t_{v}, i\right)}\left(\tau_{w}^{(i-M(h))}+\Delta(h)\right)\right) .
$$

Definition 28 (Critical Path). For the ith firing of any transition $t_{v} \in \mathcal{T}, \tau_{v}^{(i)}$, we denote any path that belongs to the arg max of the inner maximum of the right-hand side of (27) as a critical path for the firing $\tau_{v}^{(i)}$.

\section{B.2 Cycle Time}

In this section, we describe the system periodic behavior under the constraint of having a single token per directed circuit.

LEMMA 1. In an $M G$, the number of tokens in a directed circuit remains the same after any firing sequence. 
Same as Theorem 1 in Ramamoorthy and Ho [27].

LEMMA 2. In an MG-L,

$$
\hat{T}_{u}=T_{\text {sys }}, \quad \forall t_{u} \in \mathcal{T}
$$

Same as Theorem 2 in Ramamoorthy and Ho [27]; all transitions in a live and safe MG have the same cycle time, $T_{\text {sys }}$.

LEMMA 3. In an $M G-\mathrm{L}$,

$$
T_{\text {sys }} \geq \max _{C_{k} \in C}\left(\frac{\Delta\left(C_{k}\right)}{M\left(C_{k}\right)}\right)=\Delta_{M A X}
$$

The lower bound on $T_{\text {sys }}$ is well established to be the maximum delay divided by the number of tokens for every circuit in the net $[13,15,25]$. In the example of Figure $6, \Delta_{M A X}=\max (10,10 / 2,16 / 2)=10$.

Definition 29 (Critical Circuit). We denote any circuit with the $\Delta / M$ value of $\Delta_{M A X}$ as a critical circuit.

LEMMA 4. In an MG-LT,

$$
\hat{\mathcal{H}}\left(t_{u}, t_{u}, 1\right) \subset C, \forall t_{u} \in \mathcal{T} .
$$

In other words, given an MG-LT, any loop that contains one token only has to be a directed circuit.

ProOF.

- Since it is a live and safe MG, then there is no loop with zero tokens and $\hat{\mathcal{H}}\left(t_{u}, t_{u}, 0\right)=\emptyset$. Therefore, $\hat{\mathcal{H}}\left(t_{u}, t_{u}, 1\right)$ is the set of all single-token loops that start and end with $t_{u}$.

- Intuitively, a loop has to be formed by the repetition and/or concatenation of directed circuits. And given that every directed circuit has exactly one token, and that the loops in question are single tokened, then all of those loops have to be directed circuits.

Lemma 5. In an MG-LT, $\forall t_{u}, t_{v} \in \mathcal{T}$

$$
\begin{aligned}
\text { if } \exists h_{u \rightarrow v} \in \mathcal{H}\left(t_{u}, t_{v}\right) \wedge M\left(h_{u \rightarrow v}\right) & =m>1, \\
\text { then } \exists t_{w} \in \mathcal{T} \text { s.t. } \exists h_{w \rightarrow v} \in \mathcal{H}\left(t_{w}, t_{v}\right) \wedge M\left(h_{w \rightarrow v}\right) & =m-1 \wedge h_{w \rightarrow v} \sqsubset h_{u \rightarrow v} .
\end{aligned}
$$

In other words, given a MG-LT, if there exists a path that contains more than one token between any two transitions $t_{u}$ and $t_{v}$, then there exists a path from a transition $t_{w}$ to $t_{v}$ that contains $m-1$ tokens such that path is a part of the path from $t_{u}$ to $t_{v}$.

Proof.

- In an MG-LT, it is trivial to deduce $m_{n} \leq 1, \forall m_{n} \in \mathcal{M}_{0}$.

- Given the condition in (34), going in order through the elements constituting the path $h_{u \rightarrow v}$, let us focus on the first place $p_{w}$ whose $m_{w}>0$, its value has to be exactly 1 . Since this is an MG, then there exists only one transition $t_{w}$ such that $p_{w} t_{w} \in \mathcal{F}$. By definition, since $M\left(h_{u \rightarrow v}\right)=m$ and $p_{w}, t_{w} \in h_{u \rightarrow v}$, then the path $h_{w \rightarrow v}$ is a part of $h_{u \rightarrow v}$ and $M\left(h_{w \rightarrow v}\right)=$ $m-1$.

Lemma 6. In an $M G-\mathrm{LT}, \forall t_{u} \in \mathcal{T}$,

$$
\begin{array}{r}
\text { if } \mathcal{C}\left(t_{u}\right) \cap \arg \max _{C_{k} \in C}\left(\frac{\Delta\left(C_{k}\right)}{M\left(C_{k}\right)}\right) \neq \emptyset, \text { then } \forall i \geq 1, \\
\max _{h \in \hat{\mathcal{H}}\left(t_{u}, t_{u}, i\right)}\left(\tau_{u}^{(i-M(h))}+\Delta(h)\right)=\tau_{u}^{(i-1)}+\Delta_{M A X}
\end{array}
$$

In other words, given an MG-LT, for any transition $t_{u}$ that belongs to a critical circuit, the maximum dependency of the ith occurrence of $t_{u}$ on a previous transition-through a loop-cannot be higher 
than the dependency on the $(i-1)$ th occurrence along the path that is one of the critical circuits to which $t_{u}$ belong.

Proof. We prove this lemma by contradiction:

- First, let us assume that the arg max of the left-hand side of (35) cannot be a path with a single token. If $h_{x}$ with $M\left(h_{x}\right)=m_{x}>1$ does belong to that arg max, then our assumption can be written as follows:

$$
\tau_{u}^{(i-1)}+\max _{h \in \hat{\mathcal{H}}\left(t_{u}, t_{u}, 1\right)} \Delta(h)<\tau_{u}^{\left(i-m_{x}\right)}+\Delta\left(h_{x}\right) .
$$

- From Lemma 4 , every $h \in \hat{\mathcal{H}}\left(t_{u}, t_{u}, 1\right)$ is a directed circuit. Additionally, from the conditions in (35), we can then write

$$
\max _{h \in \hat{\mathcal{H}}\left(t_{u}, t_{u}, 1\right)} \Delta(h)=\max _{C_{u} \in C\left(t_{u}\right)} \Delta\left(C_{u}\right)=\Delta_{M A X}
$$

and (36) becomes

$$
\tau_{u}^{(i-1)}+\Delta_{M A X}<\tau_{u}^{\left(i-m_{x}\right)}+\Delta\left(h_{x}\right) .
$$

- From the firing rule, for the ith firing of $t_{u}$, along the path of going through the critical circuit $m_{x}$ times, we can write

$$
\tau_{u}^{(i)} \geq \tau_{u}^{\left(i-m_{x}\right)}+m_{x} \cdot \Delta_{M A X}
$$

Combining the previous two inequalities, we get

$$
\tau_{u}^{(i-1)}+\Delta_{M A X}<\tau_{u}^{(i)}-m_{x} \cdot \Delta_{M A X}+\Delta\left(h_{x}\right) .
$$

- The path $h_{x}$ is a loop by definition, and as stated in the proof of Lemma 4, a loop has to be formed by the repetition and/or concatenation of directed circuits. In addition, since $M\left(h_{x}\right)=m_{x}$, the loop $h_{x}$ has to be formed of $m_{x}$ circuits, and since $\triangle_{M A X}$ in an upper bound for circuits delay, we can write

$$
\Delta\left(h_{x}\right) \leq m_{x} \cdot \Delta_{M A X}
$$

Combining the previous two inequalities, we get

$$
\tau_{u}^{(i-1)}+\Delta_{M A X}<\tau_{u}^{(i)}-m_{x} \cdot \Delta_{M A X}+m_{x} \cdot \Delta_{M A X}
$$

- This leads to

$$
\tau_{u}^{(i)}>\tau_{u}^{(i-1)}+\Delta_{M A X}
$$

From the understanding of Theorem 3 and Lemma 4, and since $\Delta_{M A X}$ is at least as long as any other circuit, the previous inequality cannot be correct for any single-token loop leading to a contradiction. Therefore, a path with a single token has to belong indeed to the arg max of the left-hand side of (35). Then, from (36) and (37), we obtain the right-hand side of (35). 


\section{Theorem 4}

In an MG-LT, $\forall t_{u} \in \mathcal{T}$,

$$
\begin{gathered}
\text { if } C\left(t_{u}\right) \cap \arg \max _{C_{k} \in C}\left(\frac{\Delta\left(C_{k}\right)}{M\left(C_{k}\right)}\right) \neq \emptyset, \\
\text { then } \tau_{u}^{(i)}=\tau_{u}^{(i-m)}+m \cdot \Delta_{M A X}, \quad \forall i \geq m \geq 1 .
\end{gathered}
$$

In other words, given an MG-LT, if a transition $t_{u}$ is part of a critical circuit, then the $i t h$ firing of $t_{u}$ is $m$ times $\triangle_{M A X}$ its $(i-m)$ th firing. A similar result can be found in McGee and Nowick [23]. A formal proof is provided as follows.

Proof.

(a) For any $t_{u}$ that takes part in a critical circuit as formalized in (44), substituting from Theorem 3 , we get $\forall i \geq 0$

$$
\tau_{u}^{(i)}=\max _{t_{v} \in \mathcal{T}}\left(\max _{h \in \hat{\mathcal{H}}\left(t_{v}, t_{u}, i\right)}\left(\tau_{v}^{(i-M(h))}+\Delta(h)\right)\right) .
$$

(b) We prove that the arg max of the outer maximum in (45) is $t_{u}$ by contradiction.

(c) First, let us assume that it is indeed $t_{u}$.

- Substituting from Lemma 6, we can thus write

$$
\tau_{u}^{(i)}=\max _{h \in \hat{\mathcal{H}}\left(t_{u}, t_{u}, i\right)}\left(\tau_{u}^{(i-M(h))}+\Delta(h)\right)=\tau_{u}^{(i-1)}+\Delta_{M A X}, \quad \forall i \geq 1
$$

- Applying (46) recursively, we can directly deduce that for an arbitrary integer $m$,

$$
\tau_{u}^{(i)}=\tau_{u}^{(i-m)}+m \cdot \Delta_{M A X}, \quad \forall i \geq m \geq 1 .
$$

And thus we prove (44).

(d) Now we prove that the arg max of the outer maximum in (45) is $t_{u}$ by contradiction.

- For $t_{u}$ not to be the arg max of the outer maximum set in (45), this would mean that $\exists t_{x} \in \mathcal{T}, t_{x} \neq t_{u}$, and it is an arg max of the outer maximum. Substituting from (45) and (46), this means that

$$
\tau_{x}^{\left(i-M\left(h_{x \rightarrow u}\right)\right)}+\Delta\left(h_{x \rightarrow u}\right)>\tau_{u}^{(i-1)}+\Delta_{M A X} .
$$

where $h_{x \rightarrow u} \in \hat{\mathcal{H}}\left(t_{x}, t_{u}, i\right)$, and it is one of the arg max of the inner maximum in (45).

- This $M\left(h_{x \rightarrow u}\right)$ can have any arbitrary value $m$. However, if we apply Lemma 5 recursively, we find that there exists a transition $t_{y}$ such that $h_{y \rightarrow u} \sqsubset h_{x \rightarrow u}$ and that $M\left(h_{y \rightarrow u}\right)=1$.

- Therefore, for the inequality (48) to be true, the following is necessary:

$$
\tau_{y}^{(i-1)}+\Delta\left(h_{y \rightarrow u}\right)>\tau_{u}^{(i-1)}+\Delta_{M A X}
$$

- From the firing rule across the path $h_{y \rightarrow u}$, we can directly write

$$
\tau_{u}^{(i-1)} \geq \tau_{y}^{(i-2)}+\Delta\left(h_{y \rightarrow u}\right)
$$

- Combining (49) and (50), we get

$$
\tau_{y}^{(i-1)}>\tau_{y}^{(i-2)}+\Delta_{M A X}
$$


From the understanding of Theorem 3 and Lemma 4, and since $\Delta_{M A X}$ is at least as long as any other circuit, the previous inequality cannot be correct for any single-token loop leading to a contradiction.

Violating example. As discussed in Section 4, in an attempt to clarify the intuition of Theorem 4, Figure 1 shows an MG example that violates the $\mathrm{T}$ constraint; it has a directed circuit with more than one token. It has only one directed circuit, and hence every transition is part of the critical circuit. The transitions firing cannot be described using (44) because $\Delta_{M A X}=3$, but as illustrated on the right-hand side of Figure 1, the time period between successive firings of all transitions in the MG $\left(t_{1}, t_{2}\right.$, and $\left.t_{3}\right)$ oscillates between 2 and 4 .

Corollary 1. In an $M G-\mathrm{LT}, \forall t_{s} \in \mathcal{S}$,

$$
\begin{gathered}
\text { if } C\left(t_{s}\right) \cap \arg \max _{C_{k} \in C}\left(\frac{\Delta\left(C_{k}\right)}{M\left(C_{k}\right)}\right) \neq \emptyset, \\
\text { then } \tau_{s}^{(i)}=i \cdot \Delta_{M A X}, \quad \forall i \geq 0 .
\end{gathered}
$$

Proof. We prove this corollary by induction on the ith firing:

(a) In Appendix A, we chose to assign $\tau_{s}^{(0)}=0$ for any $t_{s} \in \mathcal{S}$. This is the induction basis step.

(b) We assume that the following is true, for the kth firing of $t_{s}$

$$
\tau_{s}^{(k)}=k \cdot \Delta_{M A X}
$$

(c) For the sources that take part of a critical circuit as conditioned in (52), we can apply Theorem 4 with $m=1$ to the $(k+1)$ th firing, and we get

$$
\tau_{s}^{(k+1)}=\tau_{u}^{(k)}+\Delta_{M A X}, \quad \forall i \geq 1 .
$$

(d) Substituting from (53), we get

$$
\tau_{s}^{(k+1)}=k \cdot \Delta_{M A X}+\Delta_{M A X}=(k+1) \cdot \Delta_{M A X}, \quad \forall i \geq 1 .
$$

Corollary 2. In an $M G$-LT, $\forall t_{s} \in \mathcal{S}$,

$$
\begin{gathered}
\text { if } C\left(t_{s}\right) \cap \arg \max _{C_{k} \in C}\left(\frac{\Delta\left(C_{k}\right)}{M\left(C_{k}\right)}\right) \neq \emptyset, \\
\text { then } \Gamma_{s}^{(i)}=\Delta_{M A X}, \quad \forall i \geq 0 \\
\text { and thus } \hat{T}_{n}=T_{\text {sys }}=\triangle_{M A X}, \quad \forall t_{n} \in \mathcal{T} .
\end{gathered}
$$

Proof. It comes directly without proof from Corollary 1 (see Appendix B.2) and Lemma 2.

\section{B.3 Firing Period}

In this section, we provide the proofs for key lemmas and Theorem 1.

LEMMA 7. In an MG-LS,

$$
\left|\hat{\mathcal{H}}\left(t_{s}, t_{u}, 0\right)\right| \geq 1, \quad \forall t_{u} \in \mathcal{T} .
$$

Proof. It comes directly without proof from the definition of the root of a process graph in Hulgaard et al. [15]. It is intuitive that every transition firing has to be spawned initially from a source. Therefore, in the case of a single source, there has to exist at least one token-free path from $t_{s}$ to every transition. 
LEMMA 8. In an MG-LTSC, $\forall t_{n} \in \mathcal{T}, \forall i \geq 0$,

$$
\max _{h \in \hat{\mathcal{H}}\left(t_{s}, t_{n}, i\right)}\left(\tau_{s}^{(i-M(h))}+\Delta(h)\right)=i \cdot T_{s y s}+\max _{h \in \hat{\mathcal{H}}\left(t_{s}, t_{n}, 0\right)}(\Delta(h)) .
$$

In other words, given an MG-LTSC, the delay of any critical path, with a maximum of ith tokens from the source $t_{s}$ to any transition $t_{n}$, has to equal $i$ times $T_{\text {sys }}$ in addition to the maximum token-less path delay between $t_{s}$ and $t_{n}$.

Proof. We prove this lemma by contradiction:

- Let us assume that (58) is not correct such that $M(h)=0$ is not an arg max of the maximum on the left-hand side. Basically, we are assuming that there exists a path $h_{m}$ whose $M\left(h_{m}\right)=$ $m>0$ that is longer than the path $h_{0}$ with $M\left(h_{0}\right)=0$, where $\Delta\left(h_{0}\right)=\max _{h \in \hat{\mathcal{H}}\left(t_{s}, t_{n}, 0\right)}(\Delta(h))$. Note that $h_{0}$ has to exist according to Lemma 7. Formally, our assumption is

$$
\tau_{s}^{(i-m)}+\Delta\left(h_{m}\right)>\tau_{s}^{(i)}+\Delta\left(h_{0}\right) .
$$

- Substituting from Corollary 1 and Corollary 2, we get

$$
\Delta\left(h_{m}\right)-m \cdot T_{s y s}>\Delta\left(h_{0}\right) .
$$

- Since there are no circuits with more than one token, and that an MG-L is strongly connected by definition [25], then every single-token section that belongs to the path $h_{m}$ has to be either in a distinct circuit or this section of the path is actually a whole circuit.

- Every section/whole of those circuits has to be at most as long as their circuits, the upper bound being the value $T_{\text {sys }}$.

- Therefore, the value $m \cdot T_{\text {sys }}$ has to be larger than the summation of all of those single-token sections in the path. In the extreme case of all of these sections being whole circuits, the left-hand side of (60) would only equal the token-free part.

- The right-hand side of (60) is the maximum of such token-free paths by definition, which leads to a contradiction.

- Thus, one of the arg max of the maximum in (58) has to be a path with $M(h)=0$, and this results in

$$
\max _{h \in \hat{\mathcal{H}}\left(t_{s}, t_{n}, k\right)}\left(\tau_{s}^{(k-M(h))}+\Delta(h)\right)=\tau_{s}^{(k)}+\max _{h \in \hat{\mathcal{H}}\left(t_{s}, t_{n}, 0\right)}(\Delta(h)) .
$$

- Then substituting from Corollary 1 , we obtain (58).

\section{Theorem 1}

In an MG-LTSC, $\forall t_{n} \in \mathcal{T}, \forall i \geq 0$,

$$
\tau_{n}^{(i)}=\left(\max _{h \in \hat{\mathcal{H}}\left(t_{s}, t_{n}, 0\right)} \Delta(h)\right)+i \cdot T_{\text {sys }} .
$$

Proof. We prove this theorem by induction on the ith firing:

(a) At $i=0$, we apply Theorem 3 to get

$$
\tau_{n}^{(0)}=\max _{t_{u} \in \mathcal{T}}\left(\max _{h \in \hat{\mathcal{H}}\left(t_{u}, t_{n}, 0\right)}\left(\tau_{u}^{(0)}+\Delta(h)\right)\right) .
$$


(b) Since the only initially enabled transitions are sources by definition, the first firing of $t_{n}$ has to be spawned initially from a source. From Lemma 7, such a path exists and we can write

$$
\tau_{n}^{(0)}=\max _{h \in \hat{\mathcal{H}}\left(t_{s}, t_{n}, 0\right)}\left(\tau_{s}^{(0)}+\Delta(h)\right)
$$

But $\tau_{s}^{(0)}=0$ by definition, and hence we get the induction basis step as follows

$$
\tau_{n}^{(0)}=\max _{h \in \hat{\mathcal{H}}\left(t_{s}, t_{n}, 0\right)} \Delta(h)
$$

(c) Now let us assume that the following is true at the $k t h$ firing:

$$
\tau_{n}^{(k)}=\left(\max _{h \in \hat{\mathcal{H}}\left(t_{s}, t_{n}, 0\right)} \Delta(h)\right)+k \cdot T_{\text {sys }} .
$$

(d) At the $k=1$ th firing, we use Theorem 3 to write

$$
\tau_{n}^{(k+1)}=\max _{t_{u} \in \mathcal{T}}\left(\max _{h \in \hat{\mathcal{H}}\left(t_{u}, t_{n}, k+1\right)}\left(\tau_{u}^{(k+1-M(h))}+\Delta(h)\right)\right) .
$$

(e) Similar to the proof of Theorem 4 , we prove that the arg max of the outer maximum is $t_{s}$ by contradiction.

(f) First, let us assume that it is indeed $t_{s}$. Then we can write

$$
\tau_{n}^{(k+1)}=\max _{h \in \hat{\mathcal{H}}\left(t_{s}, t_{n}, k+1\right)}\left(\tau_{s}^{(k+1-M(h))}+\Delta(h)\right) .
$$

Then, substituting from Lemma 8, we get

$$
\tau_{n}^{(k+1)}=\left(\max _{h \in \hat{\mathcal{H}}\left(t_{s}, t_{n}, 0\right)} \Delta(h)\right)+(k+1) \cdot T_{\text {sys }},
$$

which would complete the induction on the ith firing.

(g) Now we prove the assumption by contradiction:

- For $t_{s}$ not to be the arg max of the maximum in (67), this would mean that $\exists t_{x} \in \mathcal{T}, t_{x} \neq$ $t_{s}$, and it is an arg max of the maximum such that

$$
\tau_{x}^{\left(k+1-M\left(h_{x}\right)\right)}+\Delta\left(h_{x}\right)>\max _{h \in \hat{\mathcal{H}}\left(t_{s}, t_{n}, k+1\right)}\left(\tau_{s}^{(k+1-M(h))}+\Delta(h)\right),
$$

where $h_{x} \in \mathcal{H}\left(t_{x}, t_{n}, k+1\right)$, and it is one of the arg max of the maximum.

- Substituting from Lemma 8 , the inequality becomes

$$
\tau_{x}^{\left(k+1-M\left(h_{x}\right)\right)}+\Delta\left(h_{x}\right)>\left(\max _{h \in \hat{\mathcal{H}}\left(t_{s}, t_{n}, 0\right)} \Delta(h)\right)+(k+1) \cdot T_{s y s} .
$$

And let $\Delta_{0}$ denote the value of $\max _{h \in \hat{\mathcal{H}}\left(t_{s}, t_{n}, 0\right)} \Delta(h)$ for simplicity.

- Substituting from Corollary 1 , the inequality becomes

$$
\tau_{x}^{\left(k+1-M\left(h_{x}\right)\right)}+\Delta\left(h_{x}\right)>\Delta_{0}+T_{\text {sys }}+\tau_{s}^{(k)} .
$$

- From the induction hypothesis in (66), at the $k t h$ firing, the path initiated from the $\tau_{s}^{(k)}$ is at least as long as any other path including the path $h_{x}$. Therefore, also substituting from Corollary 1 , the induction hypothesis implies that

$$
\tau_{s}^{(k)}+\Delta_{0} \geq \tau_{x}^{\left(k-M\left(h_{x}\right)\right)}+\Delta\left(h_{x}\right)
$$


- Combining the two previous inequalities, we get

$$
\tau_{x}^{\left(k+1-M\left(h_{x}\right)\right)}>\tau_{x}^{\left(k-M\left(h_{x}\right)\right)}+T_{\text {sys }} .
$$

- From the understanding of Theorem 3 and Lemma 4, and since $T_{\text {sys }}$ is at least as long as any other circuit, the previous inequality cannot be correct for any single-token loop leading to a contradiction.

Corollary 3. In an MG-LTSC,

$$
\Gamma_{u}^{(i)}=T_{s y s}, \quad \forall i \geq 0, \forall t_{u} \in \mathcal{T} .
$$

Proof. It comes directly without proof from Theorem 1.

\section{B.4 Uncertainty Condition}

In this section, we provide the proofs for a key lemma and Theorem 2.

LEMMA 9. In an $M G$-LTS, $\forall t_{u} \in \mathcal{T}$,

$$
\begin{gathered}
\text { if } C\left(t_{u}\right) \cap \arg \max _{C_{k} \in C}\left(\frac{\Delta\left(C_{k}\right)}{M\left(C_{k}\right)}\right) \neq \emptyset, \\
\text { then } \tau_{u}^{(i)}=\left(\max _{h \in \hat{\mathcal{H}}\left(t_{s}, t_{u}, 0\right)} \Delta(h)\right)+i \cdot \Delta_{M A X}, \forall i \geq 0 .
\end{gathered}
$$

Proof. We prove this lemma by induction on the ith firing:

(a) Since the only initially enabled transitions are sources by definition, the first firing of $t_{u}$ has to be spawned initially from a source. From Lemma 7, such path exists and we can write

$$
\tau_{u}^{(0)}=\max _{h \in \hat{\mathcal{H}}\left(t_{s}, t_{u}, 0\right)}\left(\tau_{s}^{(0)}+\Delta(h)\right)
$$

But $\tau_{s}^{(0)}=0$ by definition, and thus we get the induction basis step.

(b) Now let us assume that the following is true at the kth firing for an arbitrary integer $k$,

$$
\tau_{u}^{(k)}=\left(\max _{h \in \hat{\mathcal{H}}\left(t_{s}, t_{u}, 0\right)} \Delta(h)\right)+k \cdot \Delta_{M A X} .
$$

(c) Applying Theorem 4 at the $(k+1)$ th firing and with $m=1$, we get

$$
\tau_{u}^{(k+1)}=\tau_{u}^{(k)}+\Delta_{M A X} .
$$

(d) Substituting from (78) into (79), we get

$$
\tau_{u}^{(k+1)}=\left(\max _{h \in \hat{\mathcal{H}}\left(t_{s}, t_{u}, 0\right)} \Delta(h)\right)+(k+1) \cdot \Delta_{M A X} .
$$

COROLlary 4. In an MG-LTS, $\forall t_{u} \in \mathcal{T}$,

$$
\begin{gathered}
\text { if } C\left(t_{u}\right) \cap \arg \max _{C_{k} \in C}\left(\frac{\Delta\left(C_{k}\right)}{M\left(C_{k}\right)}\right) \neq \emptyset, \\
\text { then } \Gamma_{u}^{(i)}=\Delta_{M A X}, \quad \forall i \geq 0 \\
\text { and thus } \hat{T}_{u}=T_{\text {sys }}=\Delta_{M A X} .
\end{gathered}
$$

Proof. It comes directly without proof from Lemma 9 and Lemma 2. 


\section{Theorem 2}

In an MG-LTS,

$$
\begin{gathered}
\text { if } C\left(t_{s}\right) \cap \arg \max _{C_{k} \in \mathcal{C}}\left(\frac{\Delta\left(C_{k}\right)}{M\left(C_{k}\right)}\right)=\emptyset, \\
\text { then } \exists i^{\star}, \exists t_{n} \in C_{n} \in C\left(t_{s}\right) \text {, and } \exists t_{u} \in \mathcal{T}, t_{u} \neq t_{s}, \\
\text { such that } \tau_{s}^{\left(i^{\star}\right)}+\max _{h \in \hat{\mathcal{H}}\left(t_{s}, t_{n}, 0\right)}(\Delta(h))<\tau_{u}^{\left(i^{\star}-M\left(h_{u \rightarrow n}\right)\right)}+\Delta\left(h_{u \rightarrow n}\right) .
\end{gathered}
$$

Proof.

(a) Applying Corollary 4 to $t_{s}$, we know that

$$
\hat{T}_{s}=\triangle_{M A X}
$$

(b) Applying Theorem 1 to the firing of $t_{s}, \forall i \geq 0$, we get

$$
\tau_{s}^{(i)}=\max _{t_{u} \in \mathcal{T}}\left(\max _{h \in \hat{\mathcal{H}}\left(t_{u}, t_{s}, i\right)}\left(\tau_{u}^{(i-M(h))}+\Delta(h)\right)\right) .
$$

We hereby prove by contradiction that the arg max of the outer maximum of (84) cannot be $t_{s}$ for every $i$.

- We first assume that the $\arg \max$ is indeed $t_{s}$ for $\forall i \geq 0$. Therefore,

$$
\tau_{s}^{(i)}=\max _{h \in \hat{\mathcal{H}}\left(t_{s}, t_{s}, i\right)}\left(\tau_{s}^{(i-M(h))}+\Delta(h)\right) .
$$

- Let $h_{x}$, with $M\left(h_{x}\right)=m_{x}$, belong to the arg max of the preceding equation. Therefore, we can write that

$$
\tau_{s}^{(i)}=\tau_{s}^{\left(i-m_{x}\right)}+\Delta\left(h_{x}\right), \quad \forall i \geq 0 .
$$

- This $h_{x}$ is a loop by definition, and as stated in the proof of Lemma 4, a loop has to be formed by the repetition and/or concatenation of directed circuits. In addition, since $M\left(h_{x}\right)=m_{x}$, the loop $h_{x}$ has to be formed of $m_{x}$ circuits. Since $\Delta_{M A X}$ is an upper bound for circuits delay, we can write that

$$
\Delta\left(h_{x}\right)<m_{x} \cdot \Delta_{M A X} .
$$

Note that the value $\Delta\left(h_{x}\right)$ is strictly less than the right-hand side because $h_{x}$ contains at least one directed circuit with $t_{s}$ in it, and from the conditions in (82), $t_{s}$ does not take part of any critical circuit.

- From the definition of the cycle time in (24) and from (83), we can write

$$
\hat{T}_{s}=\lim _{i \rightarrow \infty} \frac{\tau_{s}^{(i)}}{i}=\Delta_{M A X}
$$

- Equations (86), (87), and (88) cannot be all correct for every $i$ leading to a contradiction.

(c) Therefore, a value of $i$ has to exist where the arg max of the outer maximum of (84) cannot be $t_{s}$. And hence, $\exists i^{\star}$ and $\exists t_{u} \in \mathcal{T}, t_{u} \neq t_{s}$, and $\exists h_{u \rightarrow s} \in \hat{\mathcal{H}}\left(t_{u}, t_{s}, i^{\star}\right)$ such that

$$
\max _{h \in \hat{\mathcal{H}}\left(t_{s}, t_{s}, i^{\star}\right)}\left(\tau_{s}^{\left(i^{\star}-M(h)\right)}+\Delta(h)\right)<\tau_{u}^{\left(i^{\star}-M\left(h_{u \rightarrow s}\right)\right)}+\Delta\left(h_{u \rightarrow s}\right) .
$$

(d) Going through the elements constituting the path $h_{u \rightarrow s}$, a transition $t_{n}$ has to exist that shares a circuit with $t_{s}$ (formally, $t_{n} \in C_{n} \in C\left(t_{s}\right)$ ) because the path ends at $t_{s}$. 
(e) Since the path $h_{u \rightarrow s}$ is more critical to $\tau_{s}^{(i)}$ than $\tau_{s}^{\left(i-M\left(h_{x}\right)\right)}$ as we proved, it has to be more critical to every transition's firing in it than the paths from $\tau_{s}^{(i)}$. Otherwise, the other path would become the most critical to $t_{s}$; we already proved its contradiction. Therefore, the part $h_{u \rightarrow n} \sqsubset h_{u \rightarrow s}$ is more critical to $\tau_{n}^{(i)}$ than any path from $\tau_{s}^{(i)}$. This can be formalized as follows:

$$
\Delta\left(h_{u \rightarrow n}\right)+\tau_{u}^{\left(i^{\star}-M\left(h_{u \rightarrow n}\right)\right)}>\max _{h \in \hat{\mathcal{H}}\left(t_{s}, t_{n}, i^{\star}\right)}\left(\tau_{s}^{\left(i^{\star}-M(h)\right)}+\Delta(h)\right) .
$$

(f) Applying Lemma 7 to the preceding inequality, we can directly deduce (82).

\section{REFERENCES}

[1] Filipp Akopyan, Jun Sawada, Andrew Cassidy, Rodrigo Alvarez-Icaza, John Arthur, Paul Merolla, Nabil Imam, et al. 2015. TrueNorth: Design and tool flow of a $65 \mathrm{mw} 1$ million neuron programmable neurosynaptic chip. IEEE Transactions on Computer-Aided Design of Integrated Circuits and Systems 34, 10 (2015), 1537-1557.

[2] Manish Amde, Tomaz Felicijan, Aristeidis Efthymiou, Douglas Edwards, and Luciano Lavagno. 2005. Asynchronous on-chip networks. IEE Proceedings-Computers and Digital Techniques 152, 2 (2005), 273-283.

[3] Peter A. Beerel, Recep O. Ozdag, and Marcos Ferretti. 2010. A Designer's Guide to Asynchronous VLSI. Cambridge University Press.

[4] Jordi Cortadella, Luciano Lavagno, and Emre Tuncer. 2013. Network of tightly coupled performance monitors for determining the maximum frequency of operation of a semiconductor IC. US Patent 8,446,224.

[5] Jordi Cortadella, Marc Lupon, Alberto Moreno, Antoni Roca, and Sachin S. Sapatnekar. 2016. Ring oscillator clocks and margins. In Proceedings of the 2016 22nd IEEE International Symposium on Asynchronous Circuits and Systems (ASYNC'16). IEEE, Los Alamitos, CA, 19-26.

[6] Ali Dasdan and Rajesh K. Gupta. 1998. Faster maximum and minimum mean cycle algorithms for system-performance analysis. IEEE Transactions on Computer-Aided Design of Integrated Circuits and Systems 17, 10 (1998), 889-899.

[7] Ilana David, Ran Ginosar, and Michael Yoeli. 1992. Implementing sequential machines as self-timed circuits. IEEE Transations on Computers 41, 1 (1992), 12-17.

[8] Mike Davies, Narayan Srinivasa, Tsung-Han Lin, Gautham Chinya, Yongqiang Cao, Sri Harsha Choday, Georgios Dimou, et al. 2018. Loihi: A neuromorphic manycore processor with on-chip learning. IEEE Micro 38, 1 (2018), 82-99.

[9] Eby G. Friedman. 2001. Clock distribution networks in synchronous digital integrated circuits. Proceedings of the IEEE 89, 5 (2001), 665-692.

[10] Kris Gaj, Eby G. Friedman, and Marc J. Feldman. 1997. Timing of multi-gigahertz rapid single flux quantum digital circuits. Fournal of VLSI Signal Processing Systems for Signal, Image and Video Technology 16, 2-3 (1997), 247-276.

[11] Matthew R. Guthaus, Dennis Sylvester, and Richard B. Brown. 2008. Clock tree synthesis with data-path sensitivity matching. In Proceedings of the 2008 Asia and South Pacific Design Automation Conference. IEEE, Los Alamitos, CA, 498-503.

[12] Matthew R. Guthaus, Gustavo Wilke, and Ricardo Reis. 2013. Revisiting automated physical synthesis of highperformance clock networks. ACM Transactions on Design Automation of Electronic Systems 18, 2 (2013), 31.

[13] Wenmian Hua and Rajit Manohar. 2017. Exact timing analysis for asynchronous systems. IEEE Transactions on Computer-Aided Design of Integrated Circuits and Systems 37, 1 (2018), 203-216.

[14] Henrik Hulgaard, Steven M. Burns, Tod Amon, and Gaetano Borriello. 1994. An Algorithm for Exact Bounds on the Time Separation of Events in Concurrent Systems. Technical Report TR \#94-02-02. Department of Computer Science and Engineering, University of Washington. (Available via anonymous ftp:cs.washington.edu:tr/1994/02/UW-CSE94-02-02.PS.Z.)

[15] Henrik Hulgaard, Steven M. Burns, Tod Amon, and Gaetano Borriello. 1995. An algorithm for exact bounds on the time separation of events in concurrent systems. IEEE Transactions on Computers 44, 11 (1995), 1306-1317.

[16] ITRS. 2015. International Technology Roadmap for Semiconductors 2.0: Beyond CMOS. Available at http://www.itrs2. net/itrs-news.html.

[17] Yoshio Kameda, Stanislav Polonsky, Masaaki Maezawa, and Takashi Nanya. 1998. Primitive-level pipelining method on delay-insensitive model for RSFQ pulse-driven logic. In Proceedings of the 1998 4th International Symposium on Advanced Research in Asynchronous Circuits and Systems. IEEE, Los Alamitos, CA, 262-273.

[18] Evangelia Kasapaki, Martin Schoeberl, Rasmus Bo Sørensen, Christoph Müller, Kees Goossens, and Jens Sparsø. 2016. Argo: A real-time network-on-chip architecture with an efficient GALS implementation. IEEE Transactions on Very Large Scale Integration (VLSI) Systems 24, 2 (2016), 479-492. 
[19] Milos Krstic, Eckhard Grass, Frank K. Gürkaynak, and Pascal Vivet. 2007. Globally asynchronous, locally synchronous circuits: Overview and outlook. IEEE Design \& Test of Computers 24, 5 (2007), 430-441.

[20] D. Leu. 1984. Properties and applications of the token distance matrix of a marked graph. In Proceedings of the IEEE 1984 International Symposium on Circuits and Systems. 1381-1385.

[21] K. K. Likharev and V. K. Semenov. 1991. RSFQ logic/memory family: A new Josephson-junction technology for subterahertz-clock-frequency digital systems. IEEE Transactions on Applied Superconductivity 1, 1 (1991), 3-28.

[22] Jan Magott. 1984. Performance evaluation of concurrent systems using Petri nets. Information Processing Letters 18, 1 (1984), 7-13.

[23] Peggy B. McGee and Steven M. Nowick. 2007. An efficient algorithm for time separation of events in concurrent systems. In Proceedings of the 2007 IEEE/ACM International Conference on Computer-Aided Design. IEEE, Los Alamitos, CA, 180-187.

[24] T. Murata. 1982. Method for realizing the synchronic distance matrix of a marked graph. In Proceedings of the IEEE International Symposium on Circuits and Systems, Vol. 2. 609-612.

[25] Tadao Murata. 1989. Petri nets: Properties, analysis and applications. Proceedings of the IEEE 77, 4 (1989), 541-580.

[26] Christian D. Nielsen and Michael Kishinevsky. 1994. Performance analysis based on timing simulation. In Proceedings of the 31st Design Automation Conference. IEEE, Los Alamitos, CA, 70-76.

[27] C. V. Ramamoorthy and Gary S. Ho. 1980. Performance evaluation of asynchronous concurrent systems using Petri nets. IEEE Transactions on Software Engineering 5 (1980), 440-449.

[28] Raymond Reiter. 1968. Scheduling parallel computations. fournal of the ACM 15, 4 (1968), 590-599.

[29] Phillip J. Restle and Alina Deutsch. 1998. Designing the best clock distribution network. In Proceedings of the 1998 Symposium on VLSI Circuits. Digest of Technical Papers. IEEE, Los Alamitos, CA, 2-5.

[30] Danil Sokolov, Julian Murphy, Alexander Bystrov, and Alexandre Yakovlev. 2005. Design and analysis of dual-rail circuits for security applications. IEEE Transactions on Computers 54, 4 (2005), 449-460.

[31] Ramy N. Tadros and Peter A. Beerel. 2017. A robust and tree-free hybrid clocking technique for RSFQ circuits-CSR application. In Proceedings of the 16th International Superconductive Electronics Conference (ISEC'17).

[32] Ramy N. Tadros and Peter A. Beerel. 2018. A robust and self-adaptive clocking technique for RSFQ cicruits-The architecture. In Proceedings of the 2018 IEEE International Symposium on Circuits and Systems (ISCAS'18). IEEE, Los Alamitos, CA.

[33] Ramy N. Tadros and Peter A. Beerel. 2018. A robust and self-adaptive clocking technique for SFQ circuits. IEEE Transactions on Applied Superconductivity 28, 7 (2018), 1-11.

[34] N. Weste and D. Harris. 2011. CMOS VLSI Design: A Circuits and Systems Perspective. Addison-Wesley.

Received April 2019; revised October 2019; accepted November 2019 